Network Working Group

Request for Comments: 4935

Category: Standards Track
C. DeSanti

$\mathrm{H} . \mathrm{K}$. Vivek

K. McCloghrie

Cisco Systems

S. Gai

Nuova Systems

August 2007

\title{
Fibre Channel Fabric Configuration Server MIB
}

Status of This Memo

This document specifies an Internet standards track protocol for the Internet community, and requests discussion and suggestions for improvements. Please refer to the current edition of the "Internet Official Protocol Standards" (STD 1) for the standardization state and status of this protocol. Distribution of this memo is unlimited.

Copyright Notice

Copyright (C) The IETF Trust (2007).

Abstract

This memo defines a portion of the Management Information Base (MIB) for use with network management protocols in the Internet community. In particular, it describes managed objects for information related to the Fabric Configuration Server function of a Fibre Channel network. 
Table of Contents

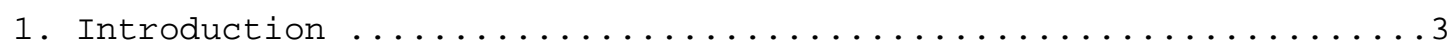

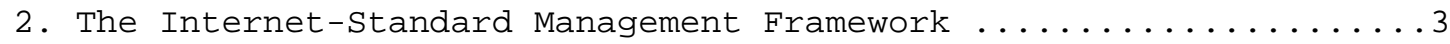

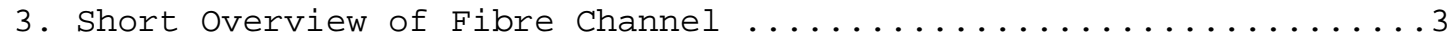

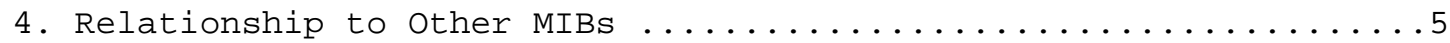

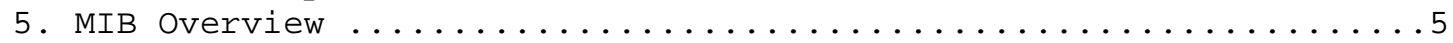

5.1. Fibre Channel Management Instance ...............

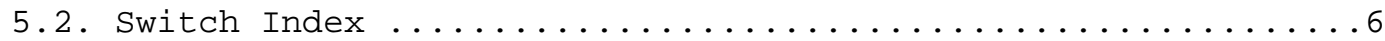

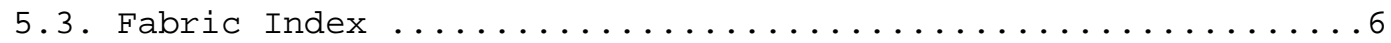

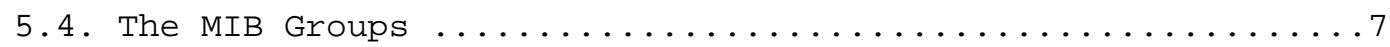

5.5. OS Logical Unit Number (LUN) Map Entries ............

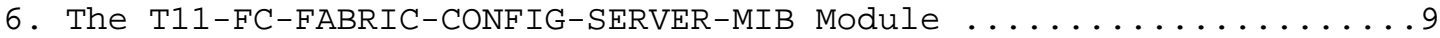

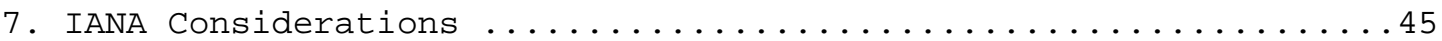

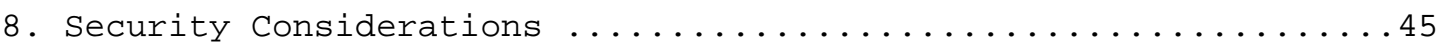

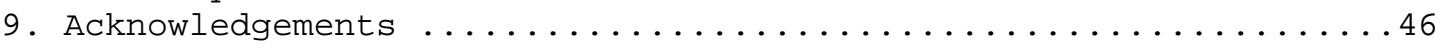

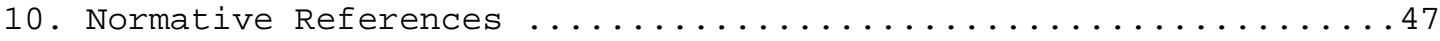

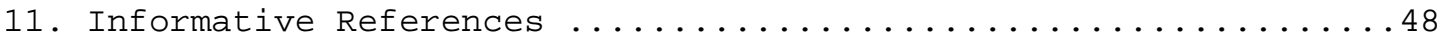




\section{Introduction}

This memo defines a portion of the Management Information Base (MIB) for use with network management protocols in the Internet community. In particular, it describes managed objects for information related to a Fibre Channel network's Fabric Configuration Server function, which provides a means by which a management application can discover Fibre Channel fabric topology and attributes. Discovered topology includes Interconnect Elements (i.e., switches, hubs, bridges, etc.) and their ports, as well as "platforms" that consist of one or more Fibre Channel nodes.

This memo was previously approved by INternational Committee for Information Technology Standards (INCITS) Task Group T11.5 (http://www.t11.org); this document is a product of the IETF's IMSS working group.

The key words "MUST", "MUST NOT", "REQUIRED", "SHALL", "SHALL NOT", "SHOULD", "SHOULD NOT", "RECOMMENDED", "MAY", and "OPTIONAL" in this document are to be interpreted as described in BCP 14, RFC 2119 [RFC2119].

2. The Internet-Standard Management Framework

For a detailed overview of the documents that describe the current Internet-Standard Management Framework, please refer to section 7 of RFC 3410 [RFC3410].

Managed objects are accessed via a virtual information store, termed the Management Information Base or MIB. MIB objects are generally accessed through the Simple Network Management Protocol (SNMP). objects in the MIB are defined using the mechanisms defined in the Structure of Management Information (SMI). This memo specifies a MIB module that is compliant to the SMIv2, which is described in STD 58, RFC 2578 [RFC2578], STD 58, RFC 2579 [RFC2579] and STD 58, RFC 2580 $[\mathrm{RFC} 2580]$.

\section{Short Overview of Fibre Channel}

The Fibre Channel (FC) is logically a bidirectional point-to-point serial data channel, structured for high performance. Fibre Channel provides a general transport vehicle for higher-level protocols such as Small Computer system Interface (SCSI) command sets, the HighPerformance Parallel Interface (HIPPI) data framing, IP (Internet Protocol), IEEE 802.2 , and others.

Physically, Fibre Channel is an interconnection of multiple communication points, called N_Ports, interconnected either by a 
switching network, called a Fabric, or by a point-to-point link. A Fibre Channel "node" consists of one or more N_Ports. A Fabric may consist of multiple Interconnect Elements, some of which are switches. An N_Port connects to the Fabric via a port on a switch called an F_Port. When multiple FC nodes are connected to a single port on a switch via an "Arbitrated Loop" topology, the switch port is called an FL_Port, and the nodes' ports are called NL_Ports. The term Nx_Port is used to refer to either an N_Port or an NL_Port. The term Fx_Port is used to refer to either an F_Port or an FL_Port. A switch port, which is interconnected to another switch port via an Inter-Switch Link (ISL), is called an E_Port. A B_Port connects a bridge device with an E_Port on a switch; a B_Port provides a subset of E_Port functionality.

Many Fibre Channel components, including the Fabric, each node, and most ports, have globally unique names. These globally unique names are typically formatted as World Wide Names (WWNs). More information on WWNs can be found in [FC-FS]. WWNs are expected to be persistent across agent and unit resets.

Fibre Channel frames contain 24-bit address identifiers that identify the frame's source and destination ports. Each FC port has both an address identifier and a WWN. When a Fabric is in use, the FC address identifiers are dynamic and are assigned by a switch. Each octet of a 24-bit address represents a level in an address hierarchy, with a Domain_ID being the highest level of the hierarchy.

The Fibre Channel Fabric Configuration Server provides a way for a management application to discover Fibre Channel fabric topology and attributes. The Fabric Configuration Server is designed so that it can be distributed among switches and accessed from any Nx_Port. However, the Fabric Configuration Server is not restricted or required to be part of/within a Fabric.

The information registered with and available from each Fabric Configuration Server is modeled as a Fabric consisting of one or more Interconnect Elements that each have some number of physical Ports, and one or more Fibre Channel nodes grouped together into Platforms to facilitate discovery and management. The Ports are connected either to other Ports on other Interconnect Elements, or to Nx_Ports. Each Interconnect Element may have attributes including its name, type, Domain Identifier, Management Identifier, Logical Name, Management Address(es), Information List, Zoning Enforcement Status, etc. Each Port may have attributes including its name, type, TX type, Module type, physical port number, attached port name(s), port state, speed, etc. Each platform may have attributes including its name, type, description, label, location, management address, etc. 
The Fibre Channel Fabric Configuration Server is defined in the FC-GS specification. The Fabric Configuration Server is one of a set of functions that are collectively known as the Management Service. The latest version of the specification is [FC-GS-5].

The latest standard for an interconnecting Fabric containing multiple Fabric Switch elements is [FC-SW-4]. [FC-SW-4] carries forward the earlier specification for the operation of a single Fabric in a physical infrastructure, and augments it with the definition of Virtual Fabrics and with the specification of how multiple Virtual Fabrics can operate within one (or more) physical infrastructures. The use of Virtual Fabrics provides for each frame to be tagged in its header to indicate which one of several Virtual Fabrics that frame is being transmitted on. All frames entering a particular "Core Switch" [FC-SW-4] (i.e., a physical switch) on the same Virtual Fabric are processed by the same "Virtual Switch" within that Core Switch.

4. Relationship to Other MIBs

The first standardized MIB for Fibre Channel [RFC2837] was focused on Fibre Channel switches. It has been replaced by the more generic Fibre Channel Management MIB [RFC4044], which defines basic information for Fibre Channel hosts and switches, including extensions to the standard IF-MIB for Fibre Channel interfaces.

This MIB extends beyond [RFC4044] to cover the functionality, in Fibre Channel switches, of providing Fibre Channel's Fabric Configuration server function.

This MIB imports some common Textual Conventions from T11-TC-MIB [RFC4439] and from T11-FC-NAME-SERVER-MIB [RFC4438]. It also imports URLString from NETWORK-SERVICES-MIB [RFC2788].

5. MIB Overview

This MIB module provides the means for monitoring the operation of, and configuring some parameters of, one or more Fabric Configuration Servers (FCS) in a Fibre Channel (FC) network. The capabilities provided include triggering a discovery of the configuration of one or more Fabrics, retrieving the results of such a discovery, as well as controlling and monitoring the operation of an FCS. The discovered configuration contains information about:

- Interconnect Elements (IEs), i.e., switches, hubs, bridges, etc.,

- Ports on IEs, and

- Platforms that consist of one or more FC nodes. 


\subsection{Fibre Channel Management Instance}

A Fibre Channel management instance is defined in [RFC4044] as a separable managed instance of Fibre Channel functionality. Fibre Channel functionality may be grouped into Fibre Channel management instances in whatever way is most convenient for the implementation(s). For example, one such grouping accommodates a single SNMP agent having multiple Agentx [RFC2741] sub-agents, with each sub-agent implementing a different Fibre Channel management instance.

The object, fcmInstanceIndex, is IMPORTed from the FC-MGMT-MIB [RFC4044] as the index value to uniquely identify each Fibre Channel management instance, for example, within the same SNMP context ([RFC3411], section 3.3.1).

\subsection{Switch Index}

The FC-MGMT-MIB [RFC4044] defines the fcmSwitchTable as a table of information about Fibre Channel switches that are managed by Fibre Channel management instances. Each Fibre Channel management instance can manage one or more Fibre Channel switches. The Switch Index, fcmSwitchIndex, is IMPORTed from the FC-MGMT-MIB as the index value to uniquely identify a Fibre Channel switch amongst those (one or more) managed by the same Fibre Channel management instance.

\subsection{Fabric Index}

With multiple Fabrics, each Fabric has its own instances of the Fabric-related management instrumentation. Thus, this MIB defines all Fabric-related information in tables that are INDEXed by an arbitrary integer, named a "Fabric Index". The syntax of a Fabric Index is T11FabricIndex, imported from T11-TC-MIB [RFC4439]. When a device is connected to a single physical Fabric, without use of any virtual Fabrics, the value of this Fabric Index will always be 1 . In an environment of multiple virtual and/or physical Fabrics, this index provides a means to distinguish one Fabric from another.

It is quite possible, and may even be likely, that a Fibre Channel switch will have ports connected to multiple virtual and/or physical Fabrics. Thus, in order to simplify a management protocol query concerning all the Fabrics to which a single switch is connected, fcmswitchIndex will be listed before t11FcsFabricIndex when they both appear in the same INDEX clause. 


\subsection{The MIB Groups}

This section describes the six MIB groups contained in the MIB module.

5.4.1. The t11FcsDiscoveredConfigGroup Group

This group contains the Fabric configuration information discovered by Fabric Configuration Servers.

5.4.2. The t11FcsDiscoverystatusGroup Group

This group contains objects by which to monitor the status of discovery of Fabric configurations by Fabric Configuration Servers.

5.4.3. The t11FcsDiscoveryControlGroup Group

This group contains objects for requesting a Fabric Configuration Server to discover the configuration of one or more Fabrics.

5.4.4. The t11FcsStatisticsGroup Group

This group contains objects for Fabric Configuration Server statistics information.

\subsubsection{The t11FcsNotificationGroup Group}

This group contains three notifications, generated when an FCS:

- rejects a registration, deregistration, or query request;

- completes discovery on a range of Fabrics;

- learns that a management address of an Interconnect Element has changed.

5.4.5.1. Flow Control for Notifications

When defining SNMP notifications for events that occur in the dataplane, the maximum frequency of their generation needs to be considered. Unless there is some limiting factor, such notifications need to be flow-controlled in some way, e.g., defined such that after some maximum number within a specified time interval have occurred, further notifications are suppressed for some subsequent time interval. However, as and when such a suppression occurs, the Network Management System (NMS) that didn't receive the notifications (because they were suppressed) needs to be able to obtain an indication of how many were suppressed. Therefore, an additional Counter32 object needs to be defined, and/or a new type of notification needs to be defined for use at the end of the interval. 
While this is extra complexity, it is necessary for notifications that need to be flow-controlled.

In contrast, for notifications such as all the ones defined in this MIB module, which are generated due to control-plane events (and are not able to start a chain reaction):

- estimating the maximum number that could possibly be generated per unit time for each type of notification is too simplistic. For example, it's unreasonable to ask how many of the t11FcsDiscoveryCompleteNotify notifications can be generated in a time interval, because it depends on several factors: how big is the network? how many Virtual Fabrics need to be discovered? how quickly can the operator ask for another discovery after the last one completes?

- the extra complexity of flow-controlling these types of notifications is not warranted.

5.4.6. The t11FcsNotificationInfoGroup Group

This group contains notification control and notification information objects for monitoring Fabric Configuration server request rejection and discovery of topology information.

\subsection{OS Logical Unit Number (LUN) Map Entries}

A "Platform" is defined in FC-GS-5 to be not only a set of zero or more FC nodes, but also a set of zero or more "OS LUN Map Entries" (see Figure 8 in [FC-GS-5]). Information on "OS LUN Map Entries" is not included in this T11-FC-FABRIC-CONFIG-SERVER-MIB. Instead, information on LUN Maps can be obtained via the scsilunMapgroup object group defined in the SCSI-MIB [RFC4455]. 
6. The T11-FC-FABRIC-CONFIG-SERVER-MIB Module

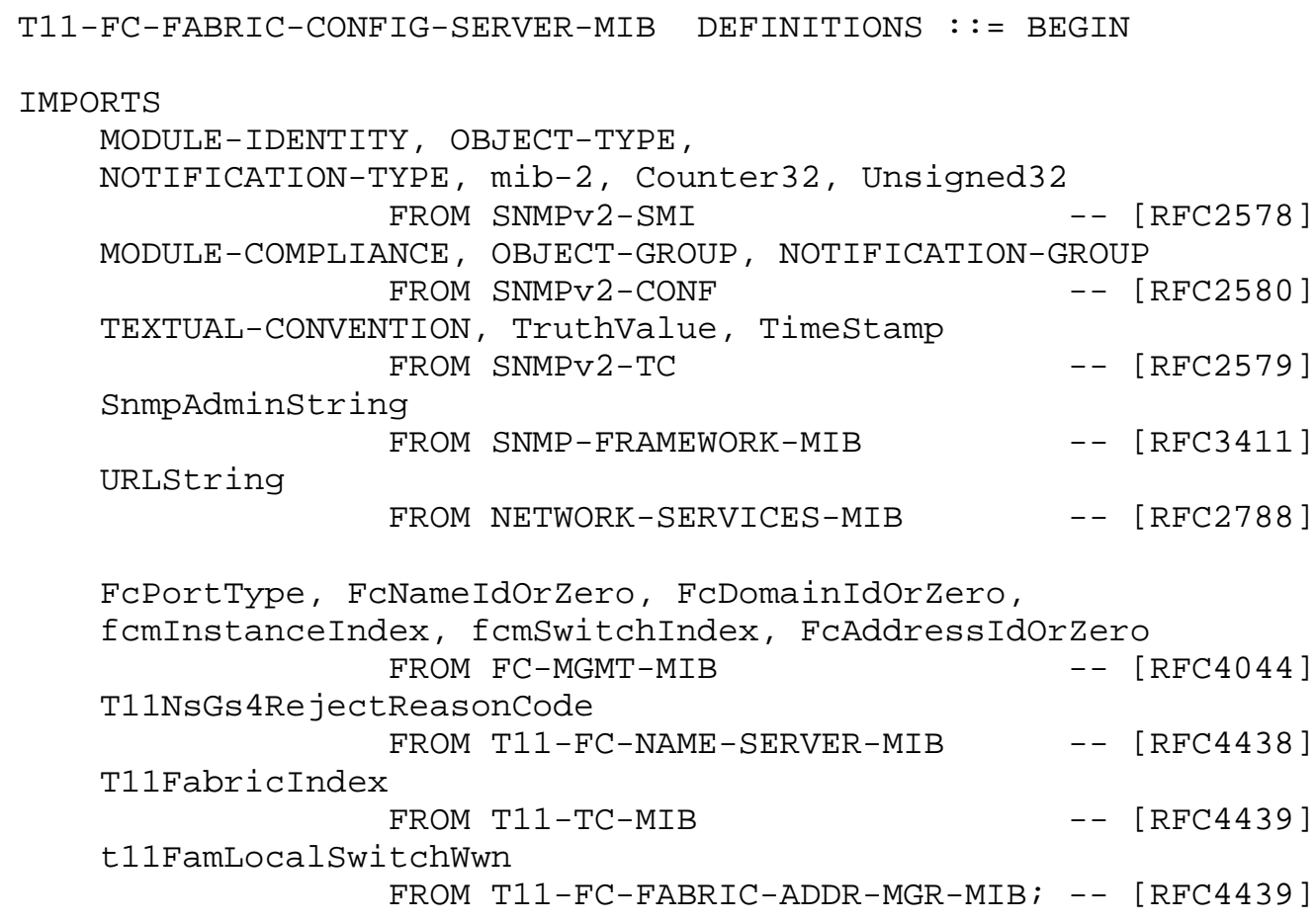

"The MIB module for the management of a Fabric Configuration Server (FCS) in a Fibre Channel (FC) network. An FCS is defined by the FC-GS-5 standard. This 


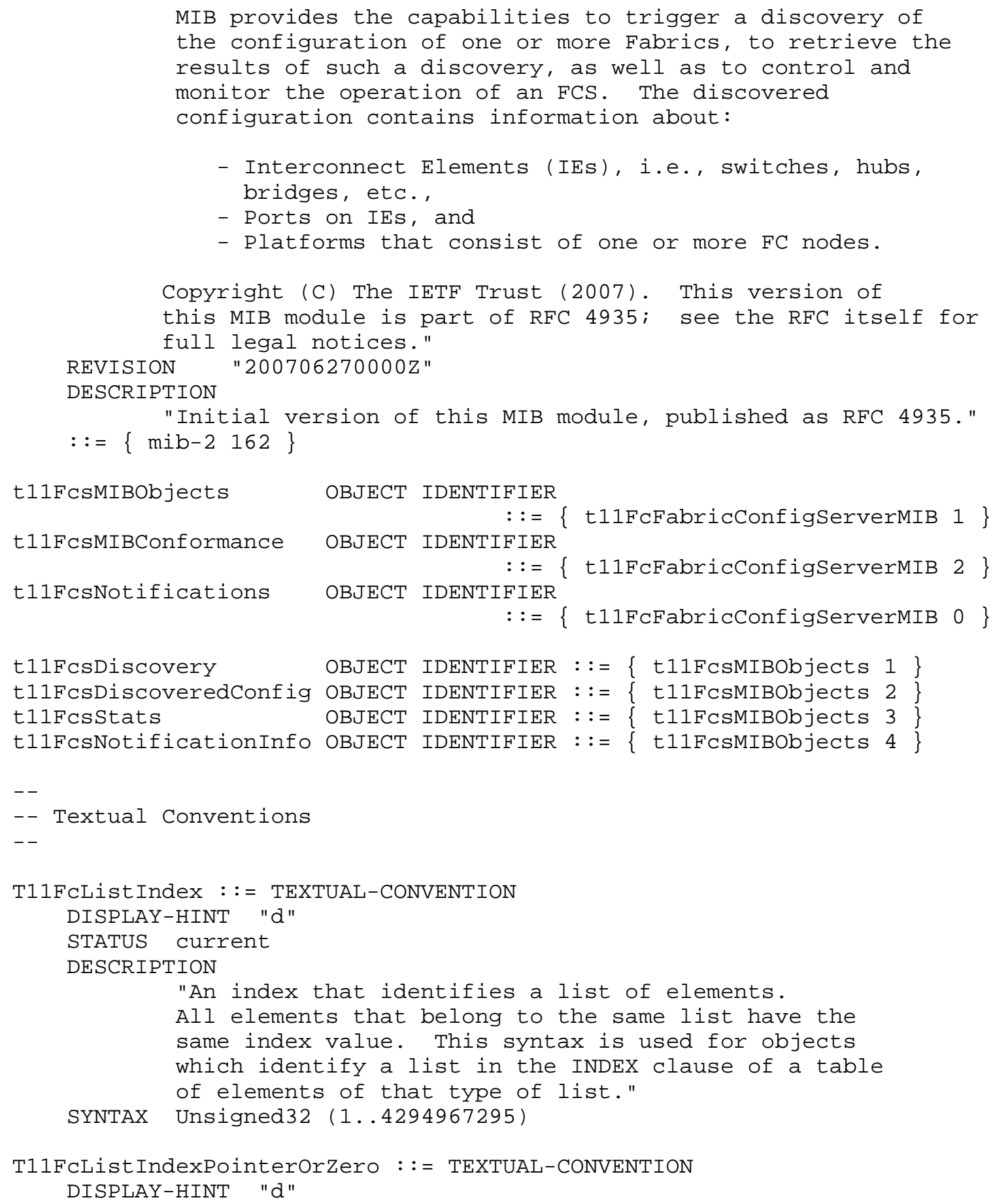




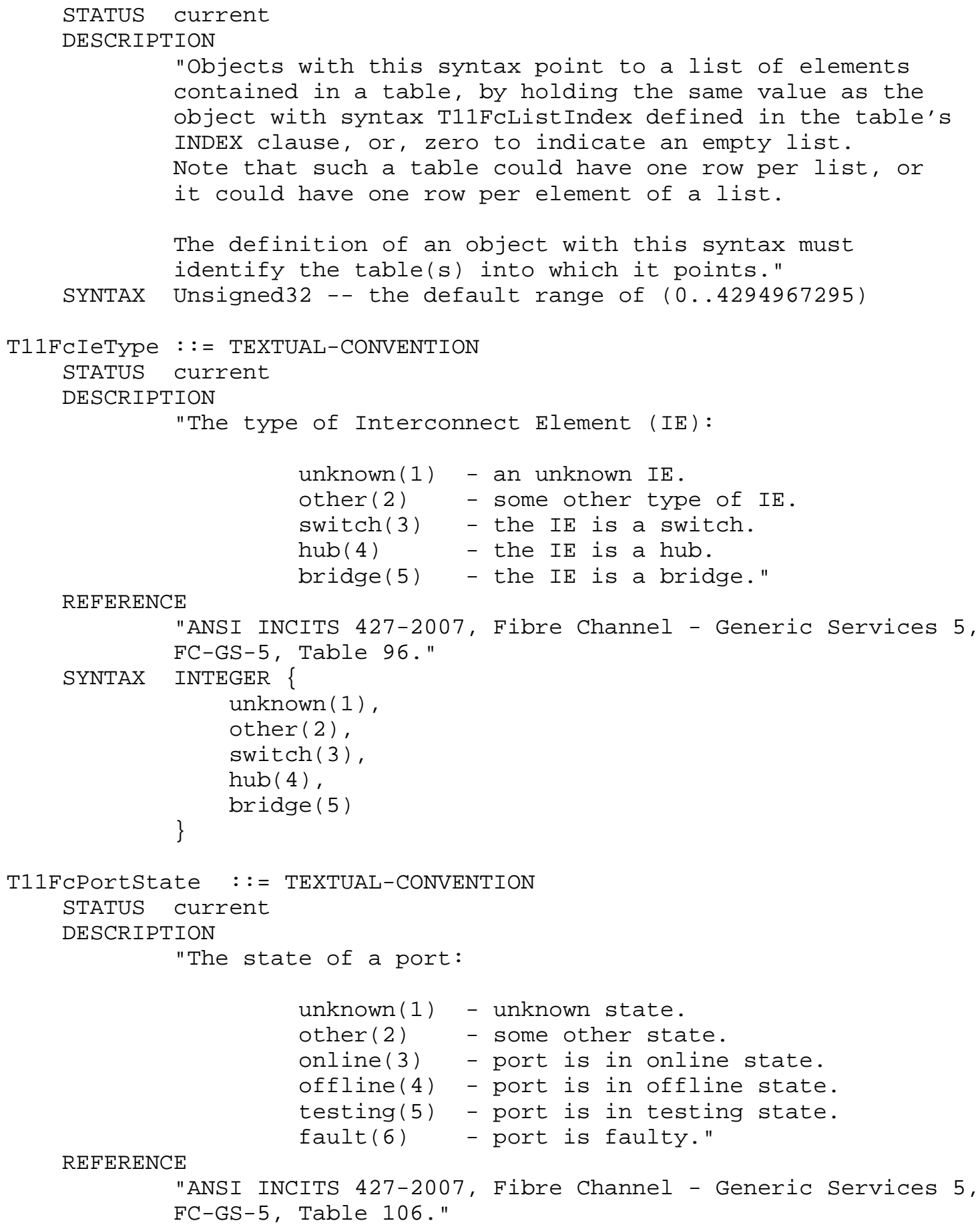




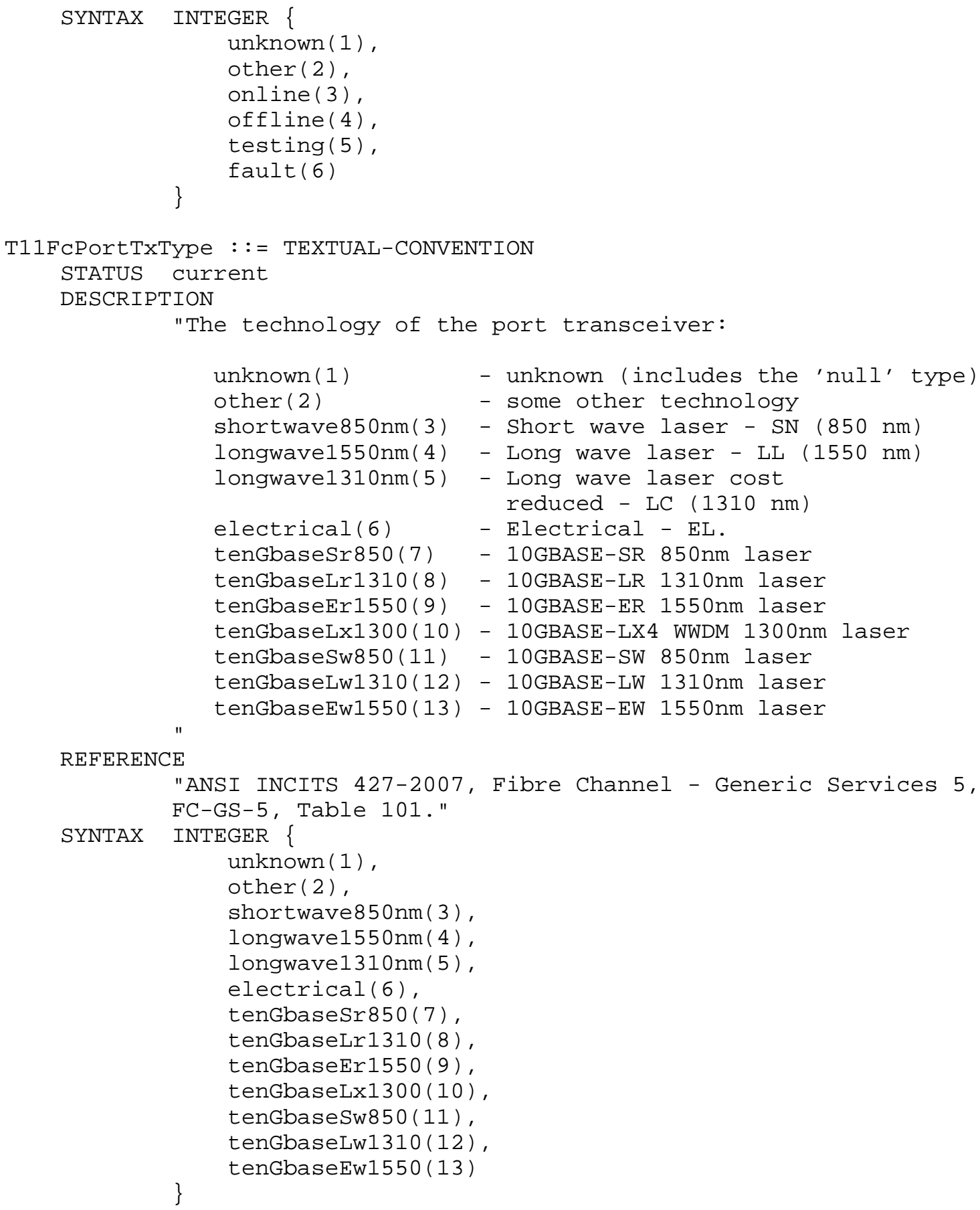


T11FcsRejectReasonExplanation : := TEXTUAL-CONVENTION

STATUS current

DESCRIPTION

"The reject reason code explanation:

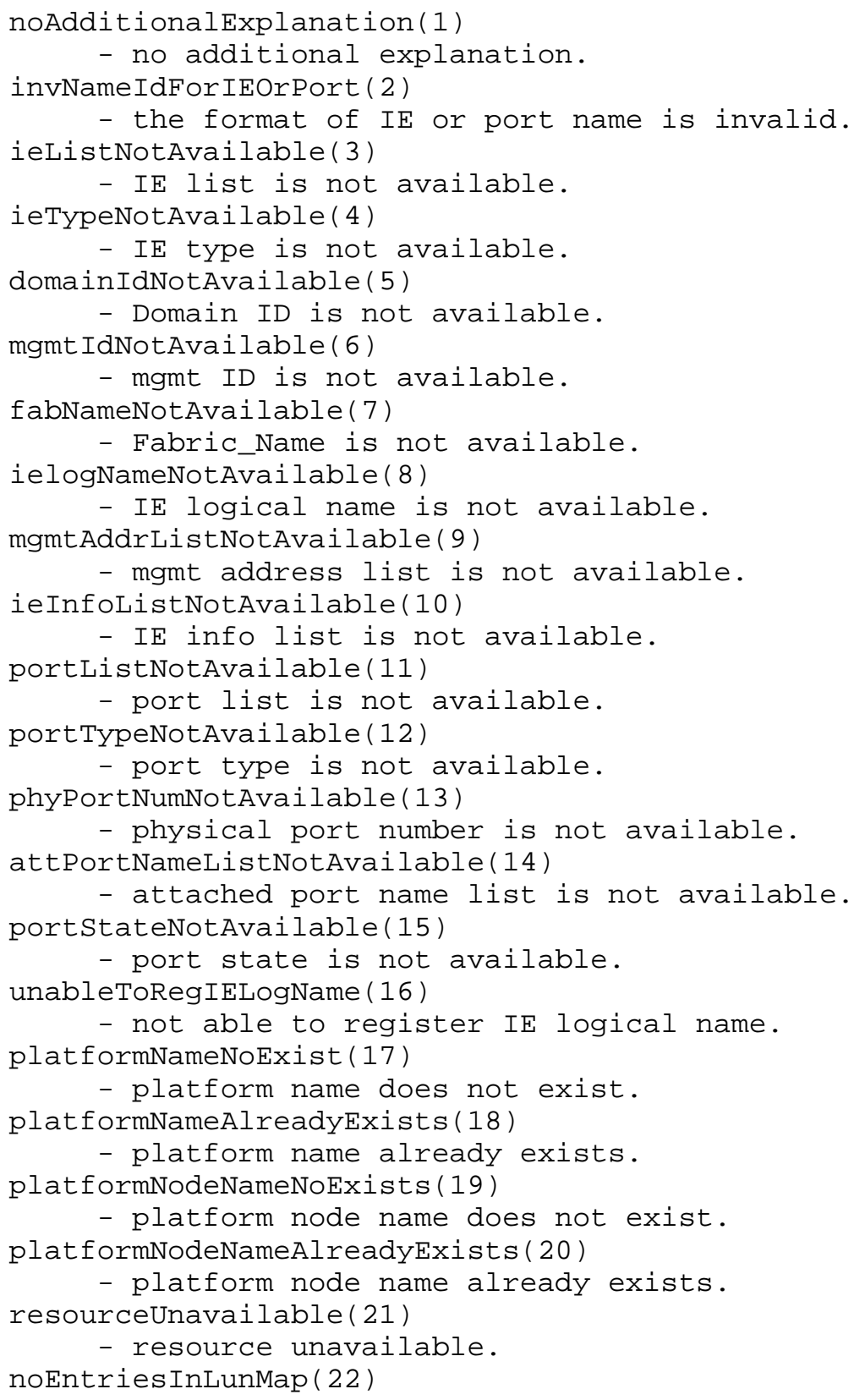




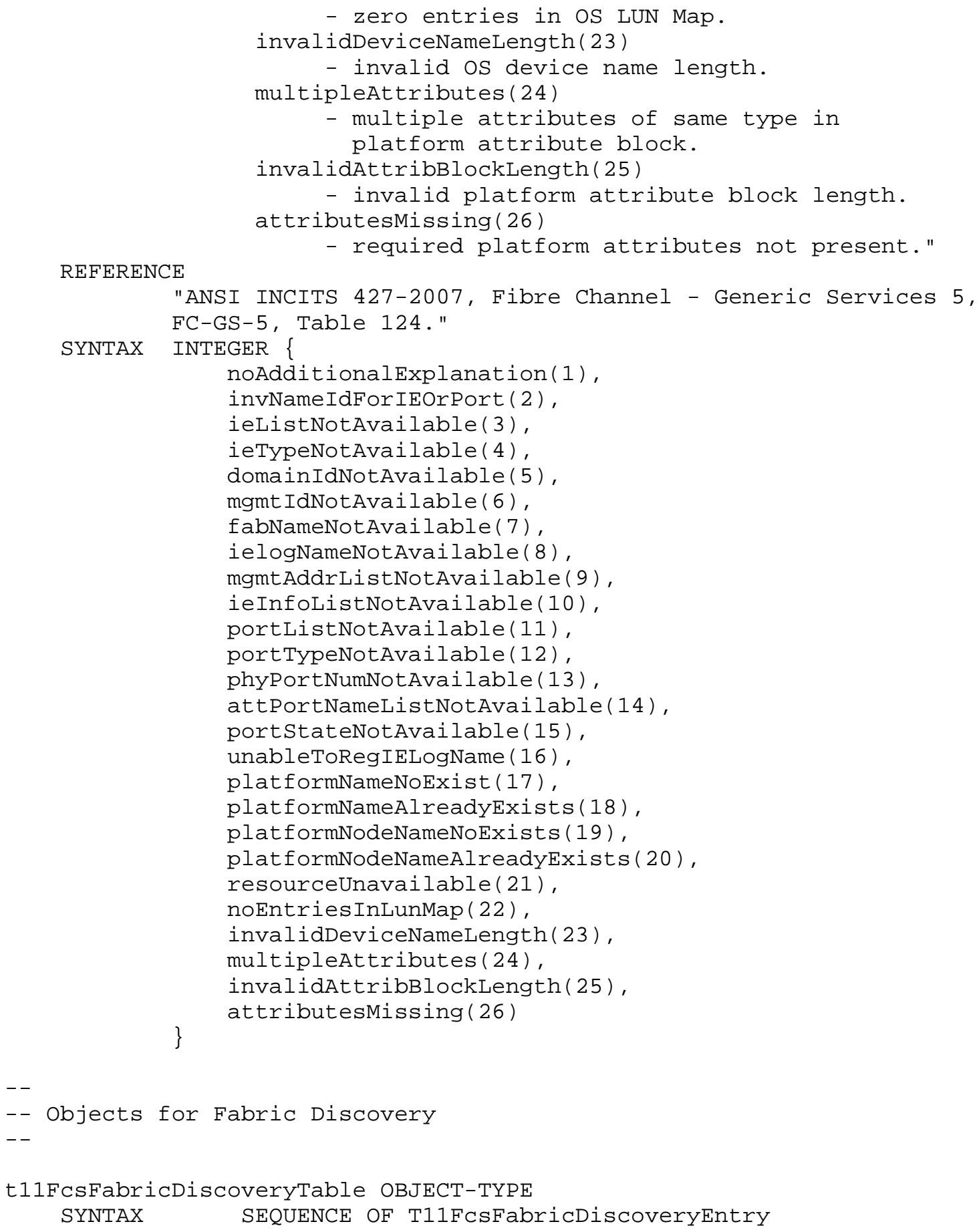




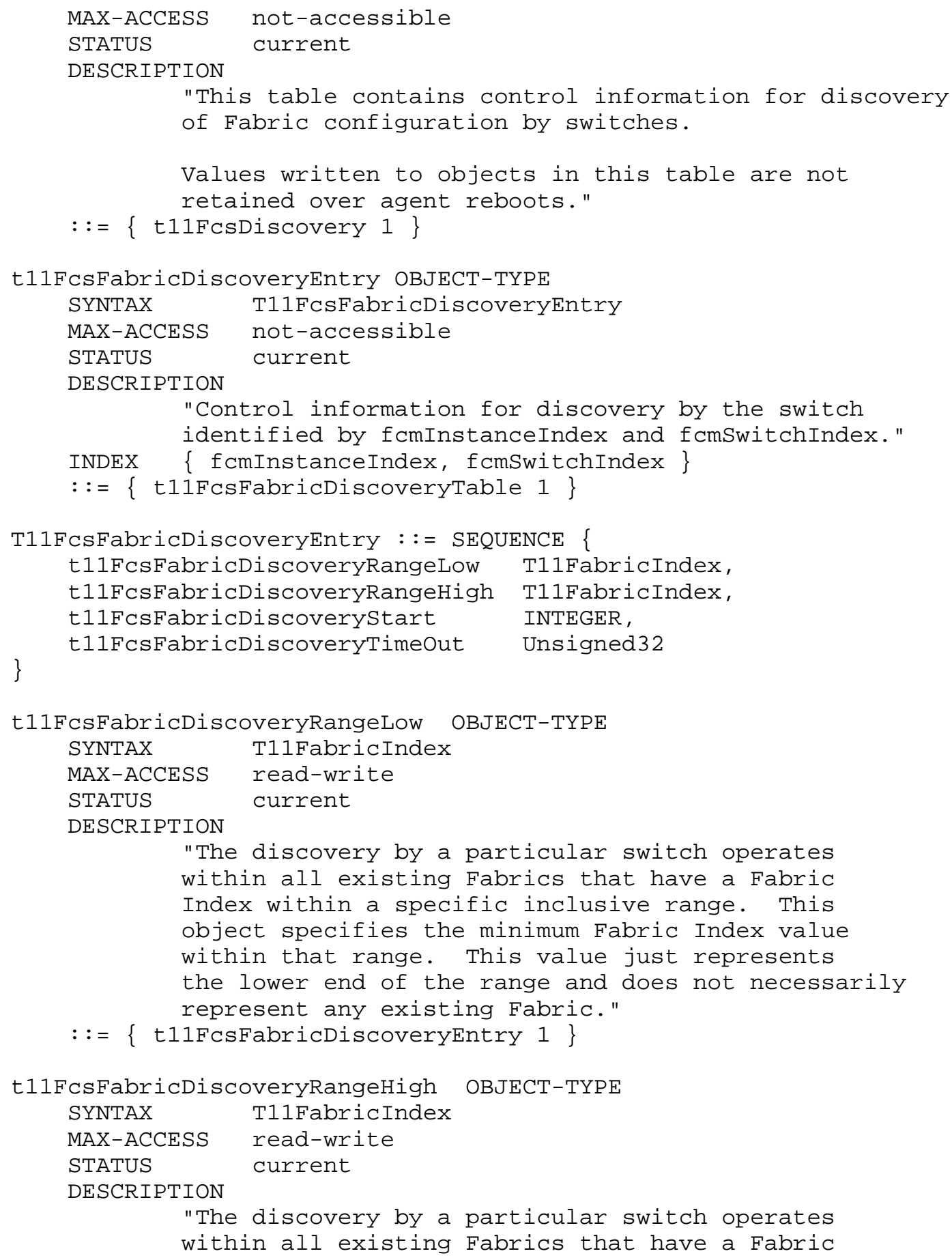




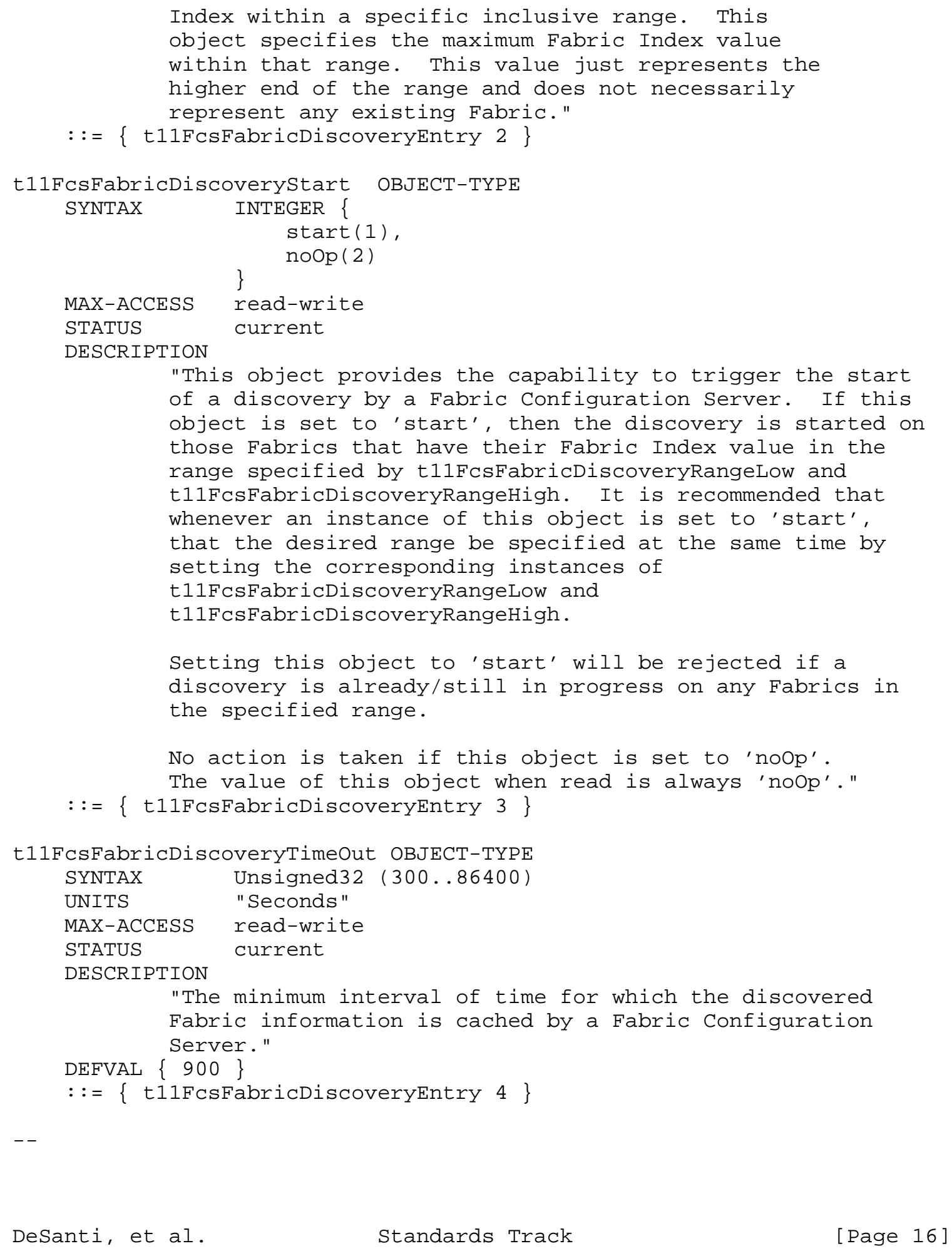




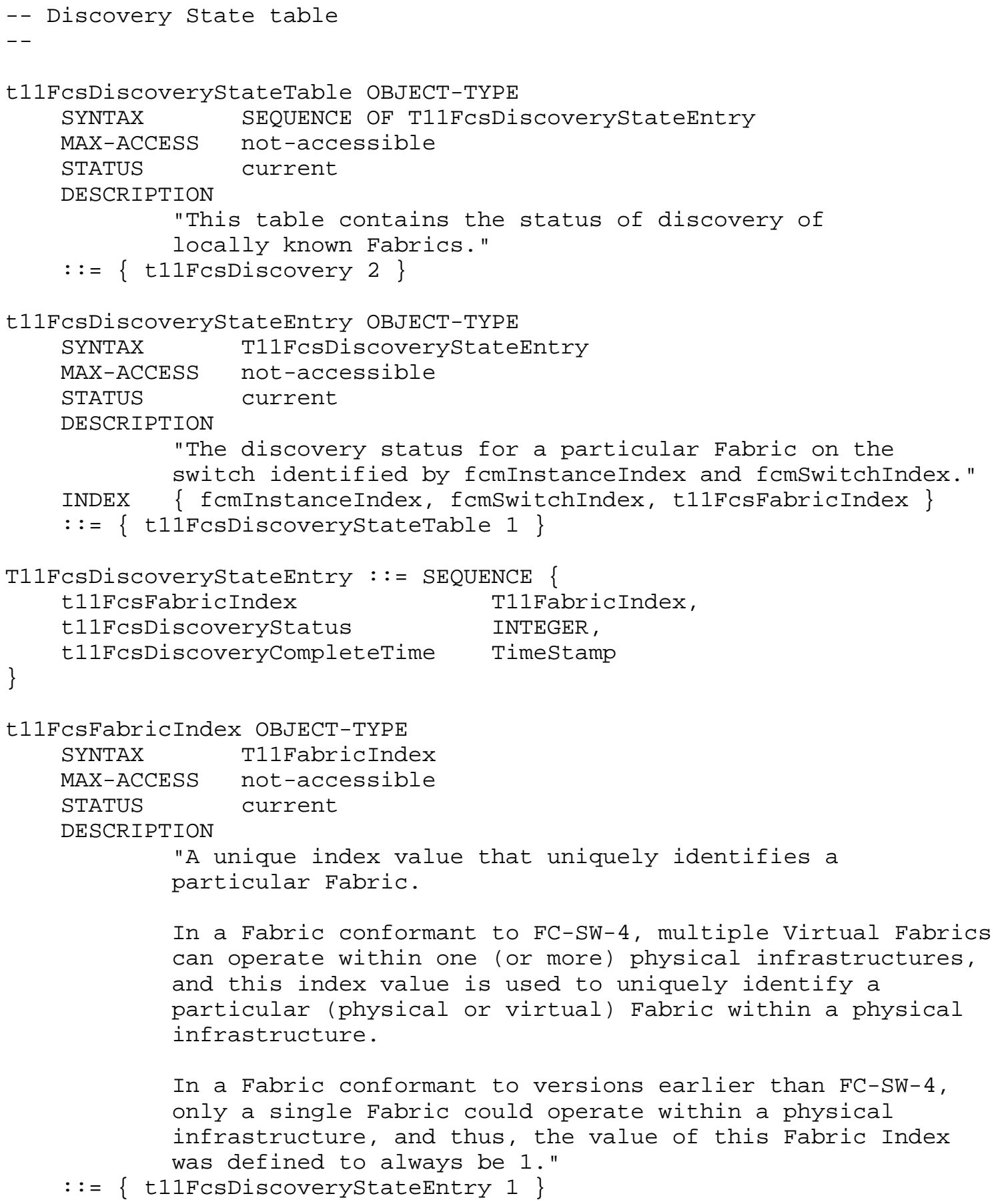




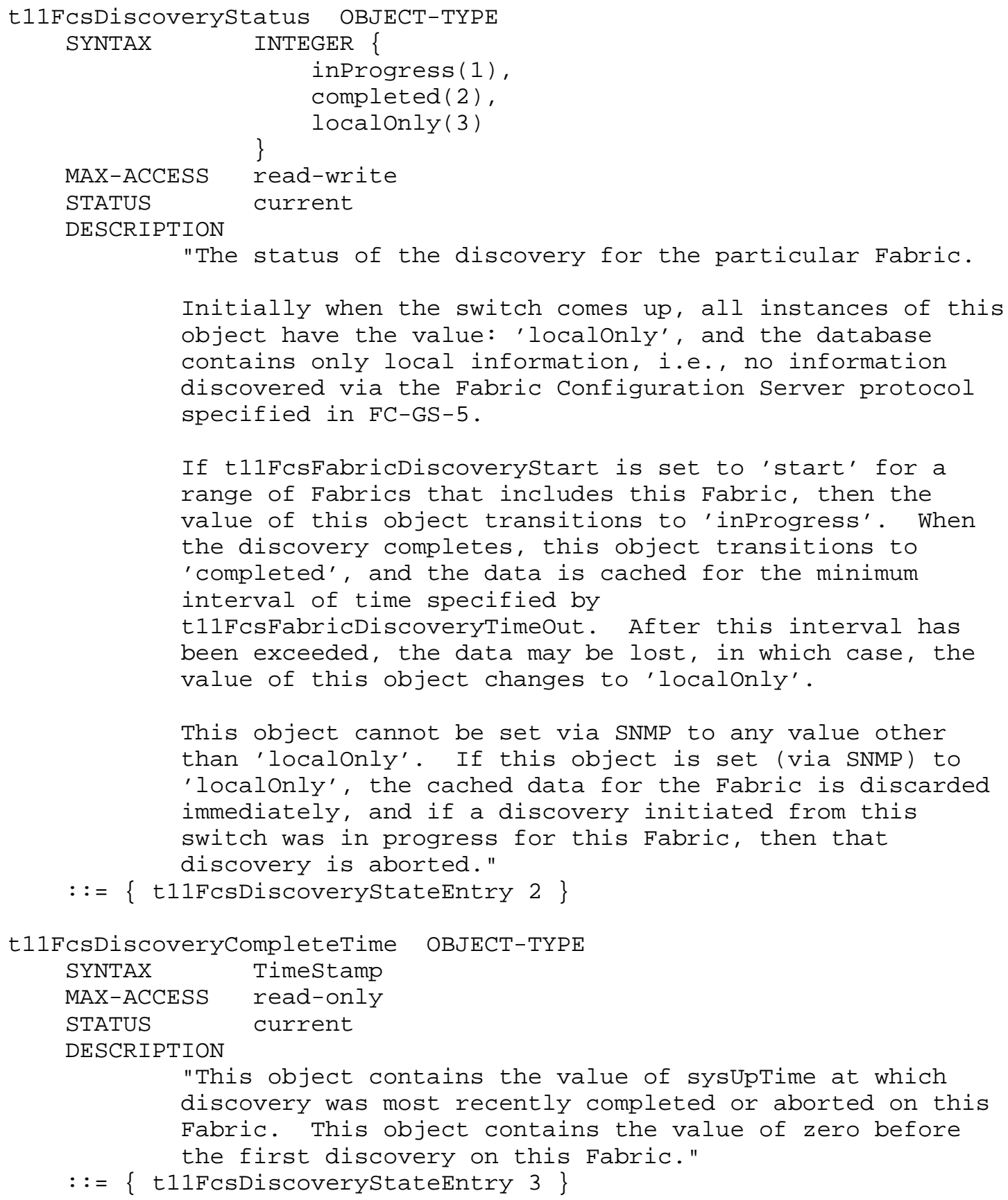




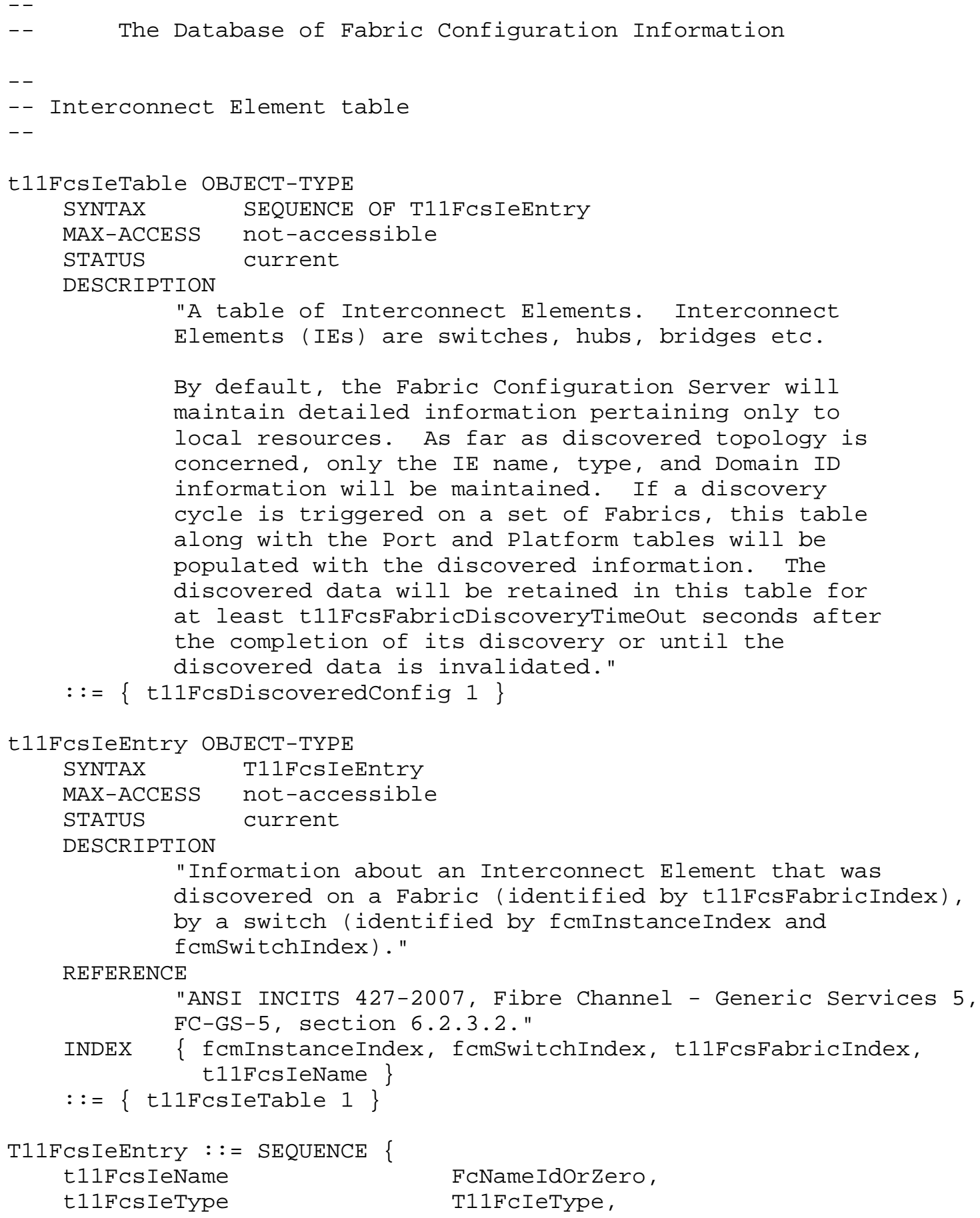




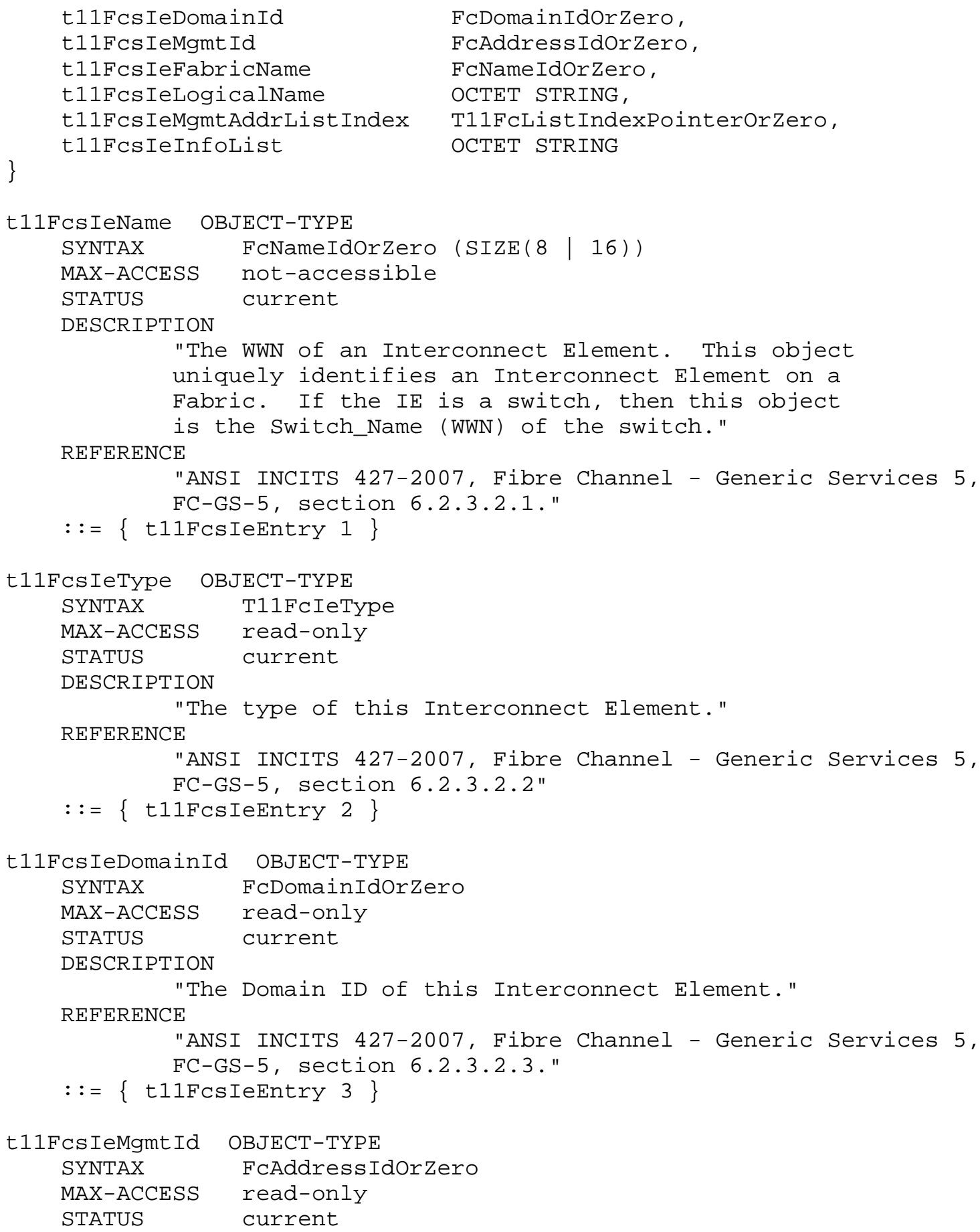




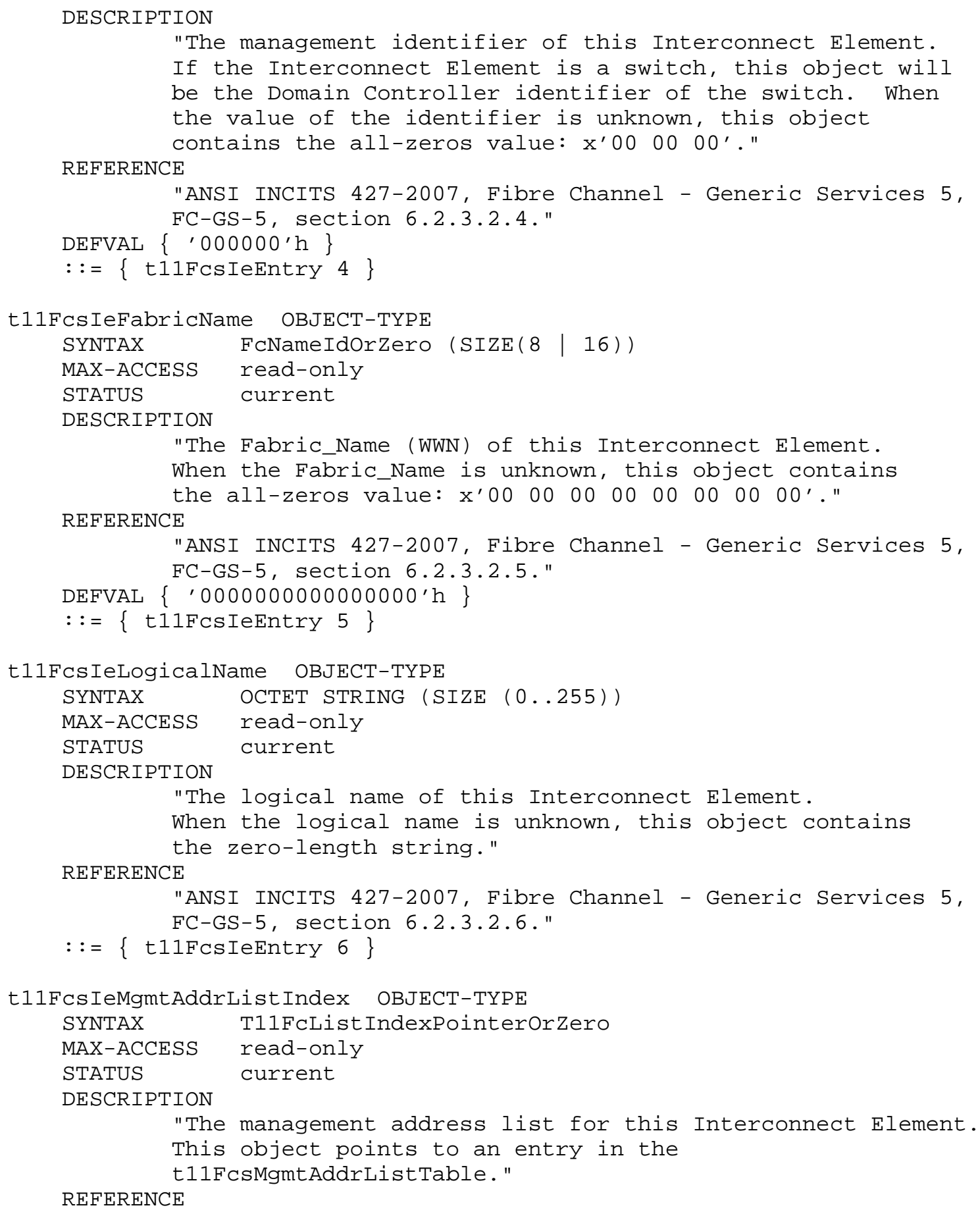




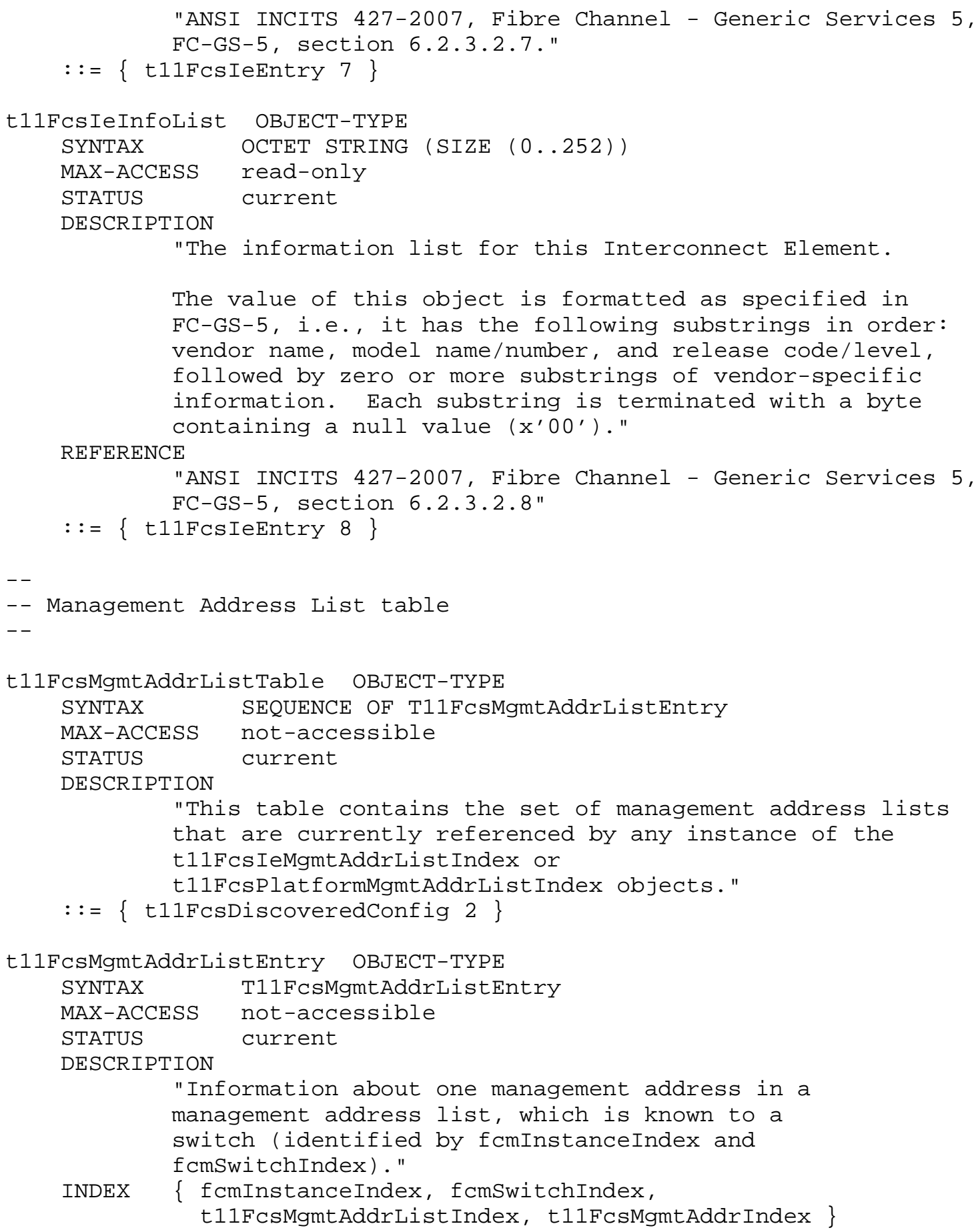


$::=\{$ t11FcsMgmtAddrListTable 1$\}$

T11FCsMgmtAddrListEntry ::= SEQUENCE \{

t11FcsMgmtAddrListIndex T11FcListIndex,

t11FcsMgmtAddrIndex Unsigned32,

\}

t11FcsMgmtAddr URLString

t11FCsMgmtAddrListIndex OBJECT-TYPE

SYNTAX TIIFCListIndex

MAX-ACCESS not-accessible

STATUS current

DESCRIPTION

"The index value of the management address list." $::=\{$ t11FcsMgmtAddrListEntry 1$\}$

t11FCsMgmtAddrIndex OBJECT-TYPE

SYNTAX Unsigned32 (1..4294967295)

MAX-ACCESS not-accessible

STATUS current

DESCRIPTION

"An integer value to distinguish different

management addresses in the same list."

$::=\{$ t11FcsMgmtAddrListEntry 2$\}$

t11FCsMgmtAddr OBJECT-TYPE

SYNTAX URLString

MAX-ACCESS read-only

STATUS current

DESCRIPTION

"The management address of this entry.

The format of this object is a Uniform Resource

Locator (URL), e.g., for SNMP, see RFC 4088."

REFERENCE

"ANSI INCITS 427-2007, Fibre Channel - Generic Services 5, FC-GS-5, section 6.2.3.2.7"

$::=\{$ t11FcsMgmtAddrListEntry 3$\}$

$--$

-- Ports

$--$

t11FcsPortTable

SYNTAX

OBJECT-TYPE

MAX-ACCESS

STATUS

SEQUENCE OF T11FCSPortEntry

DESCRIPTION

not-accessible

current

DeSanti, et al.

Standards Track

[Page 23] 


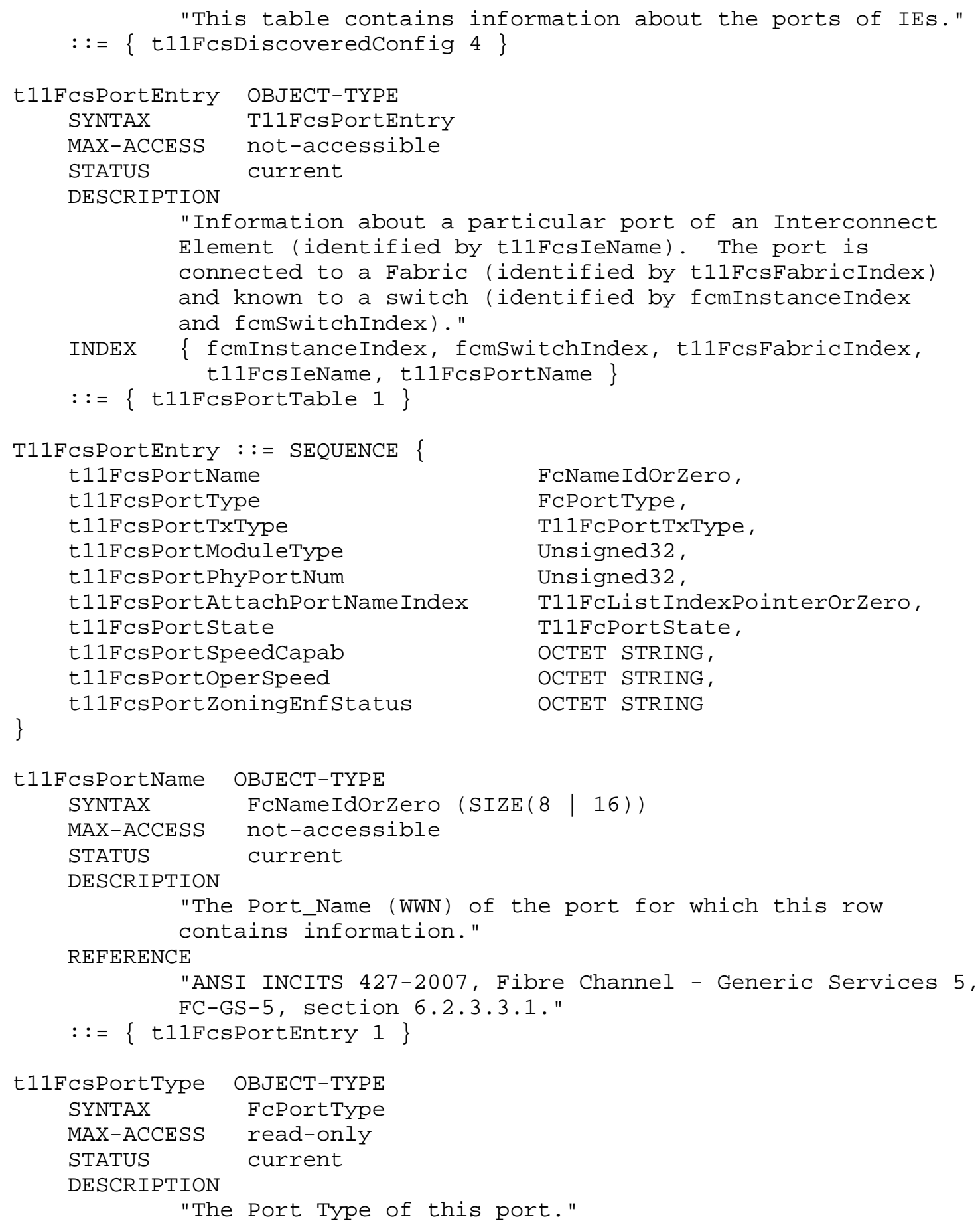




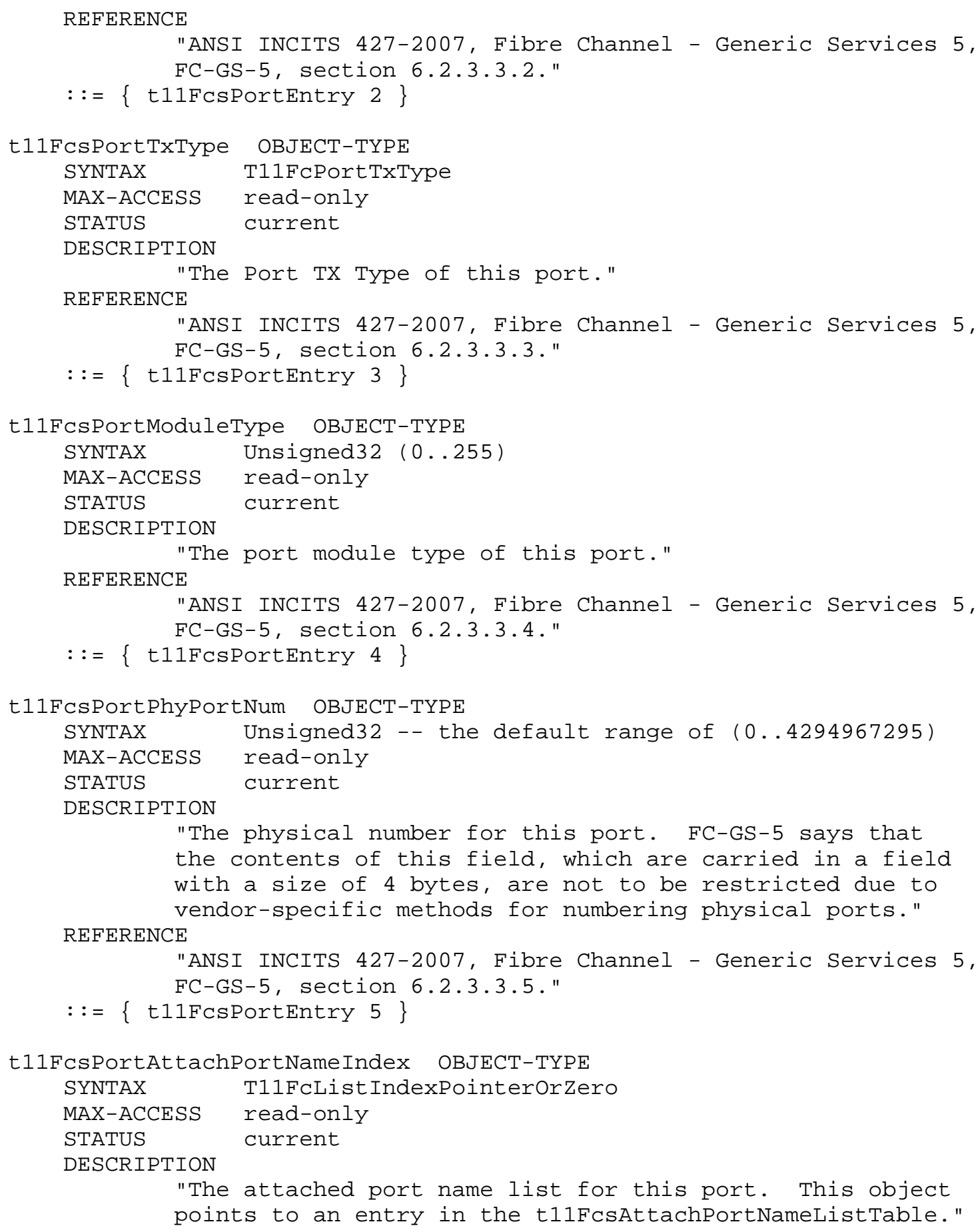




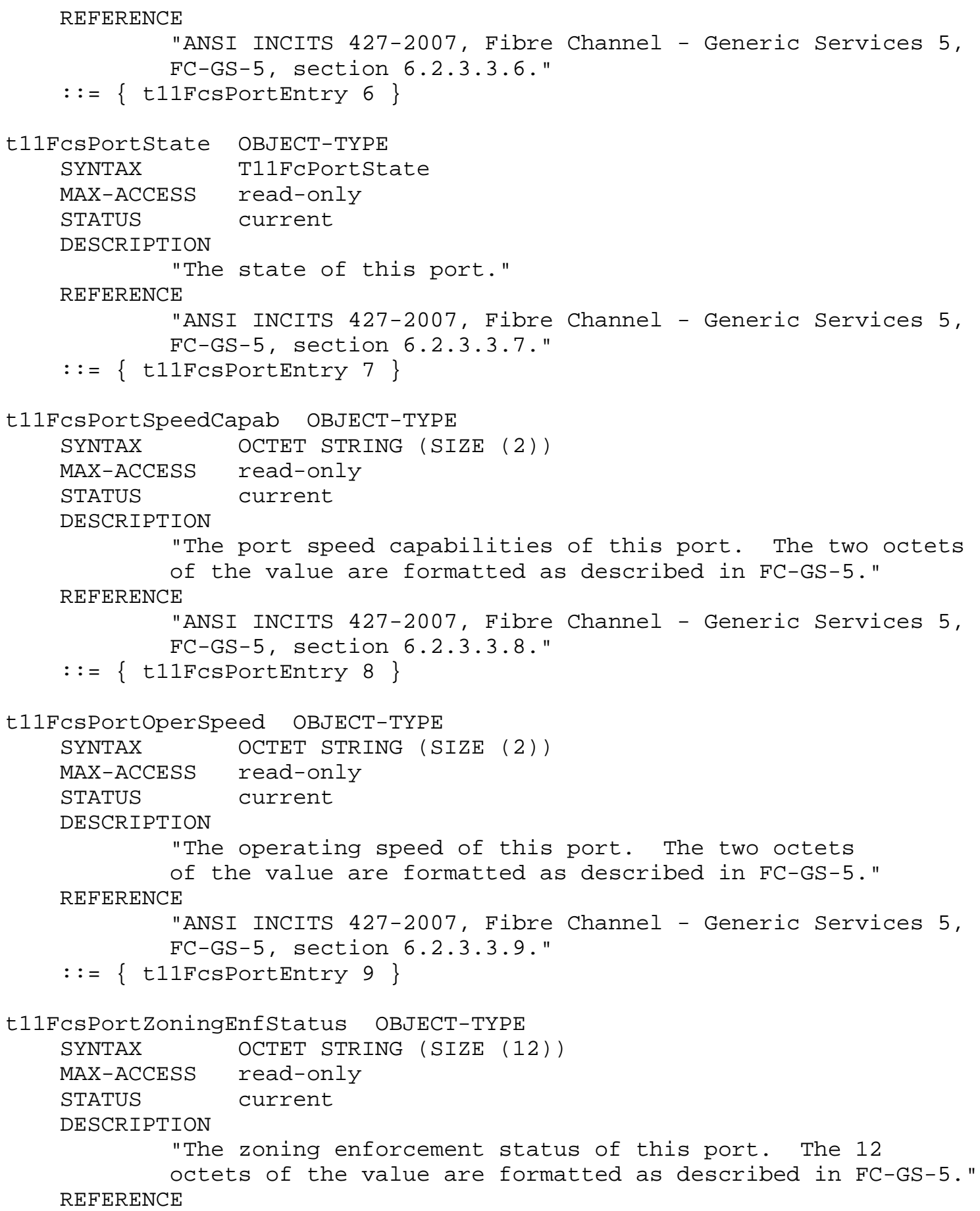




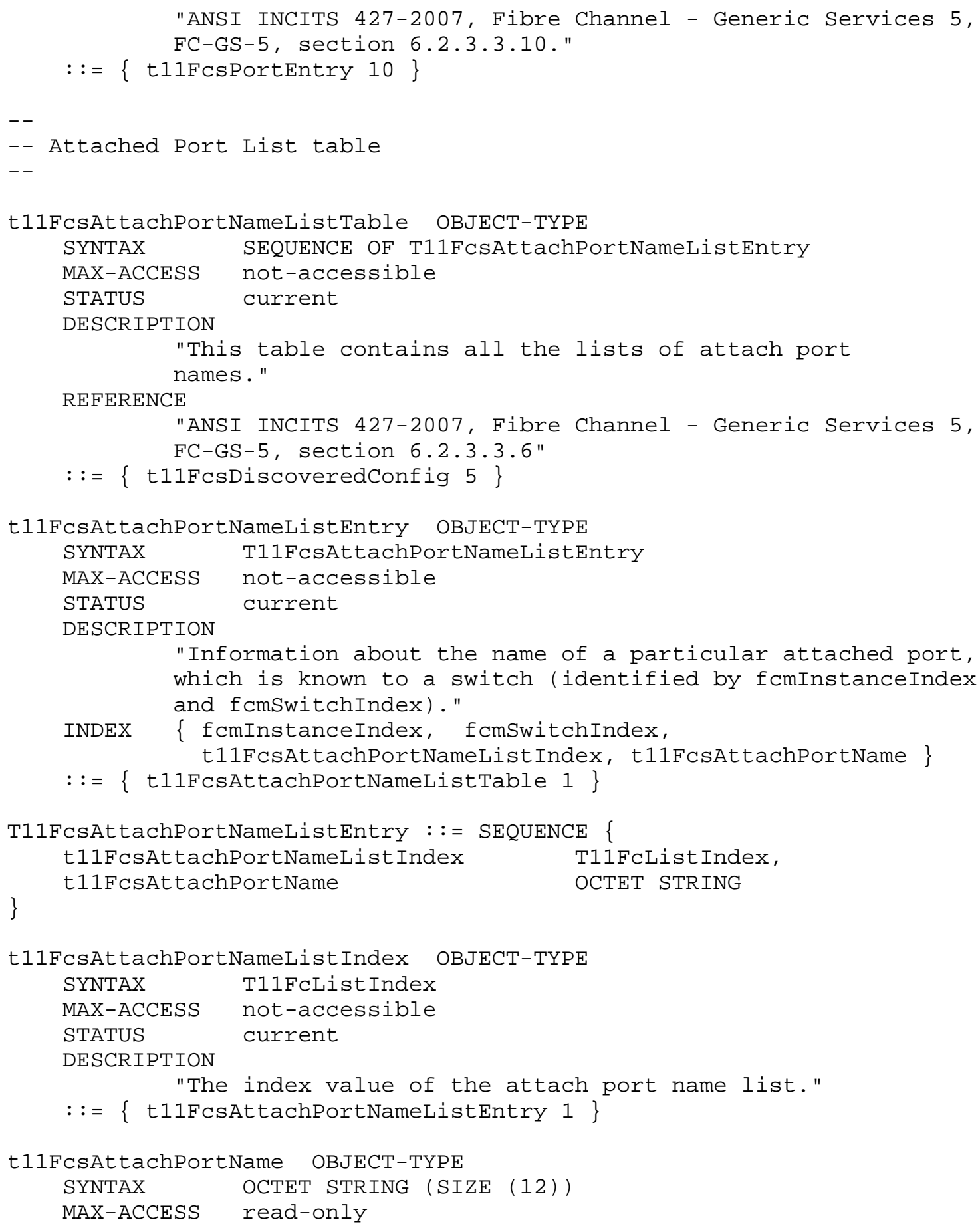




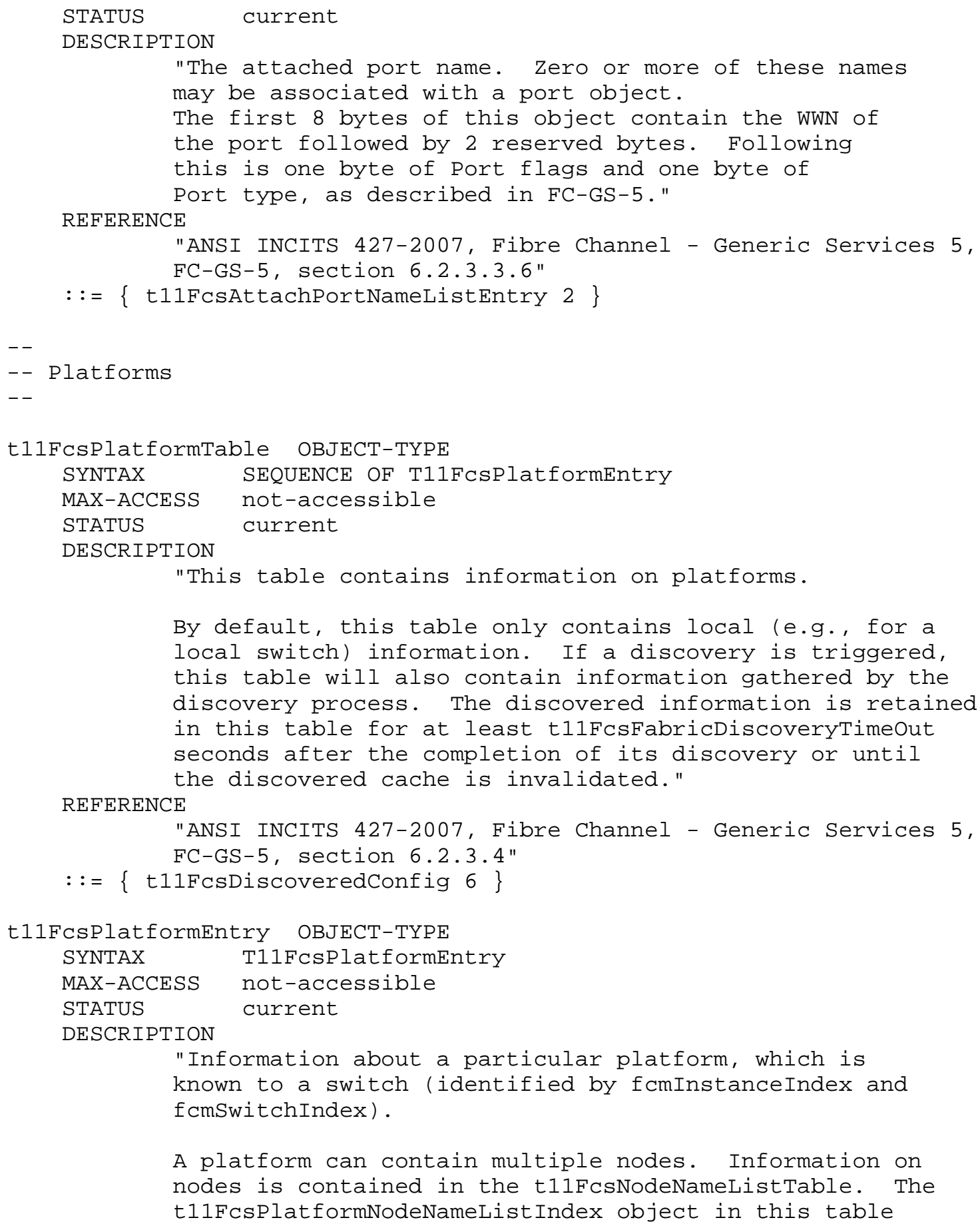




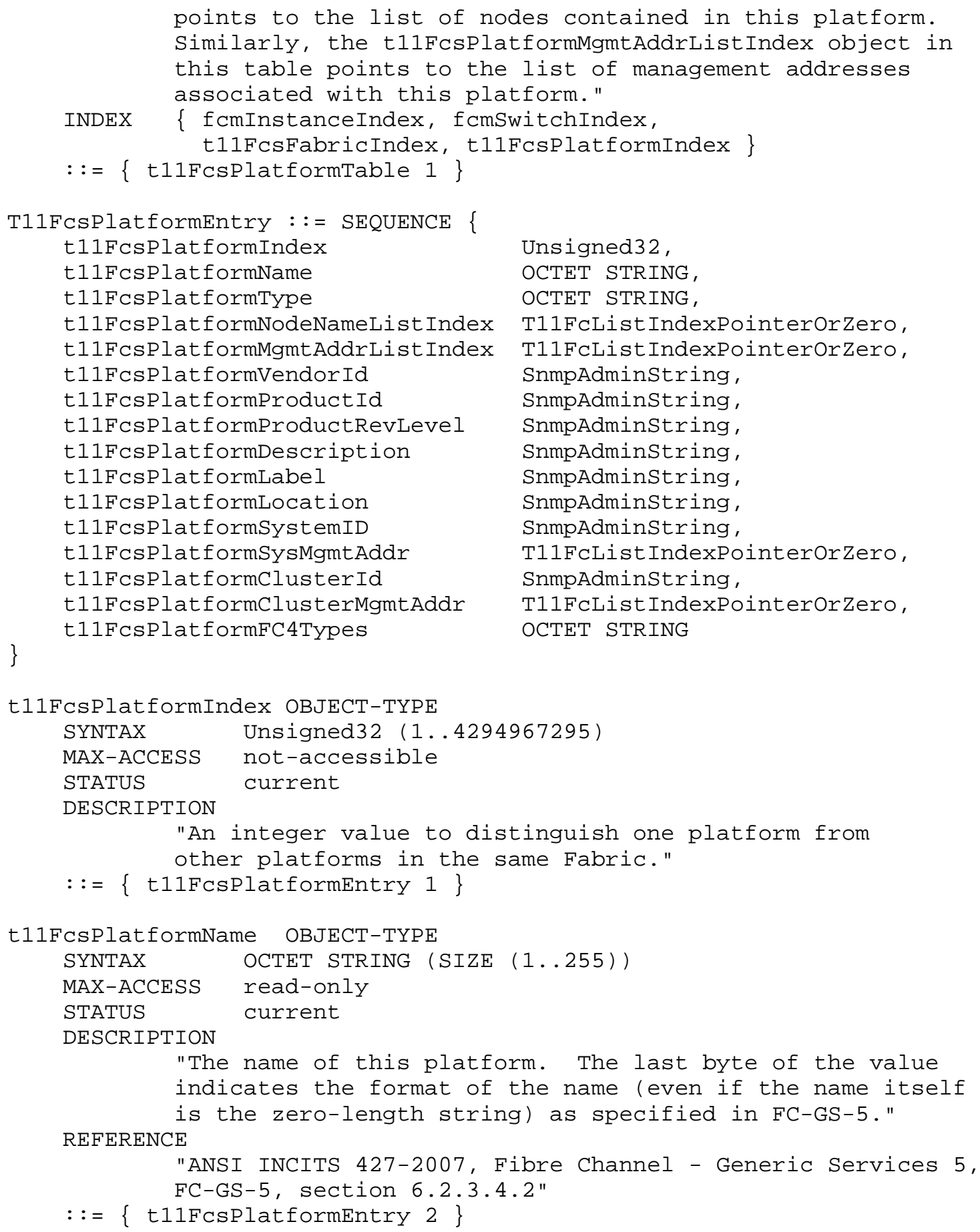




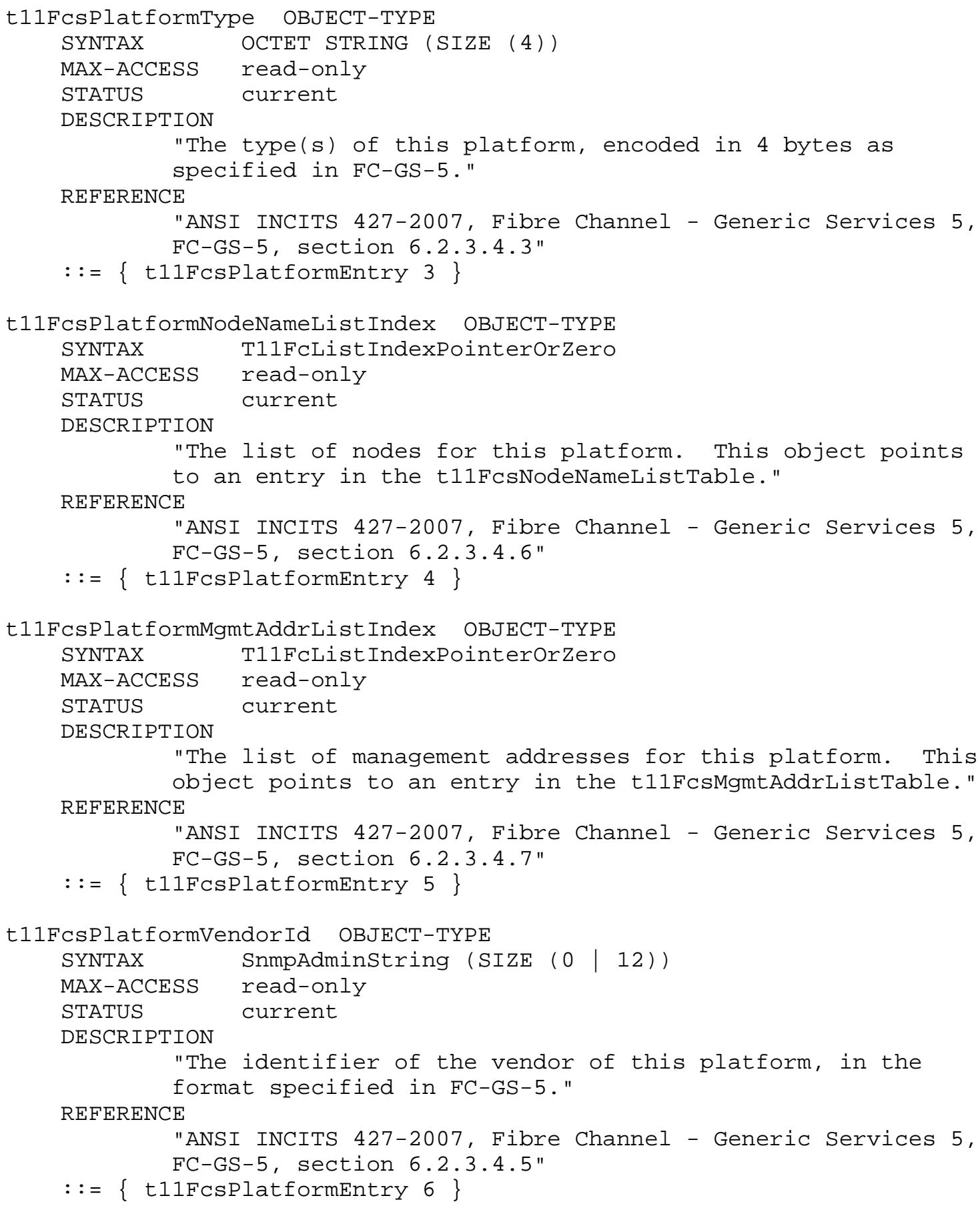




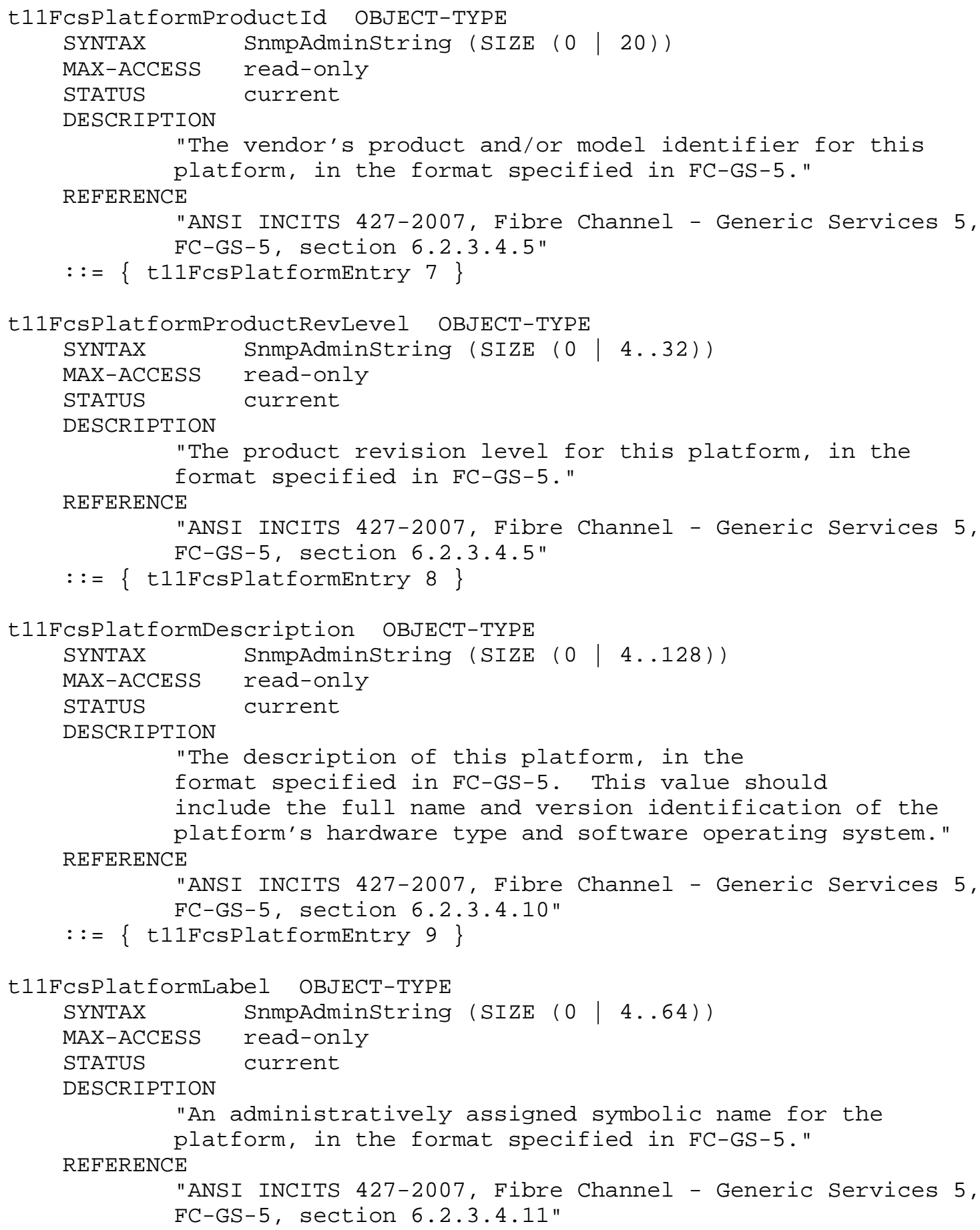




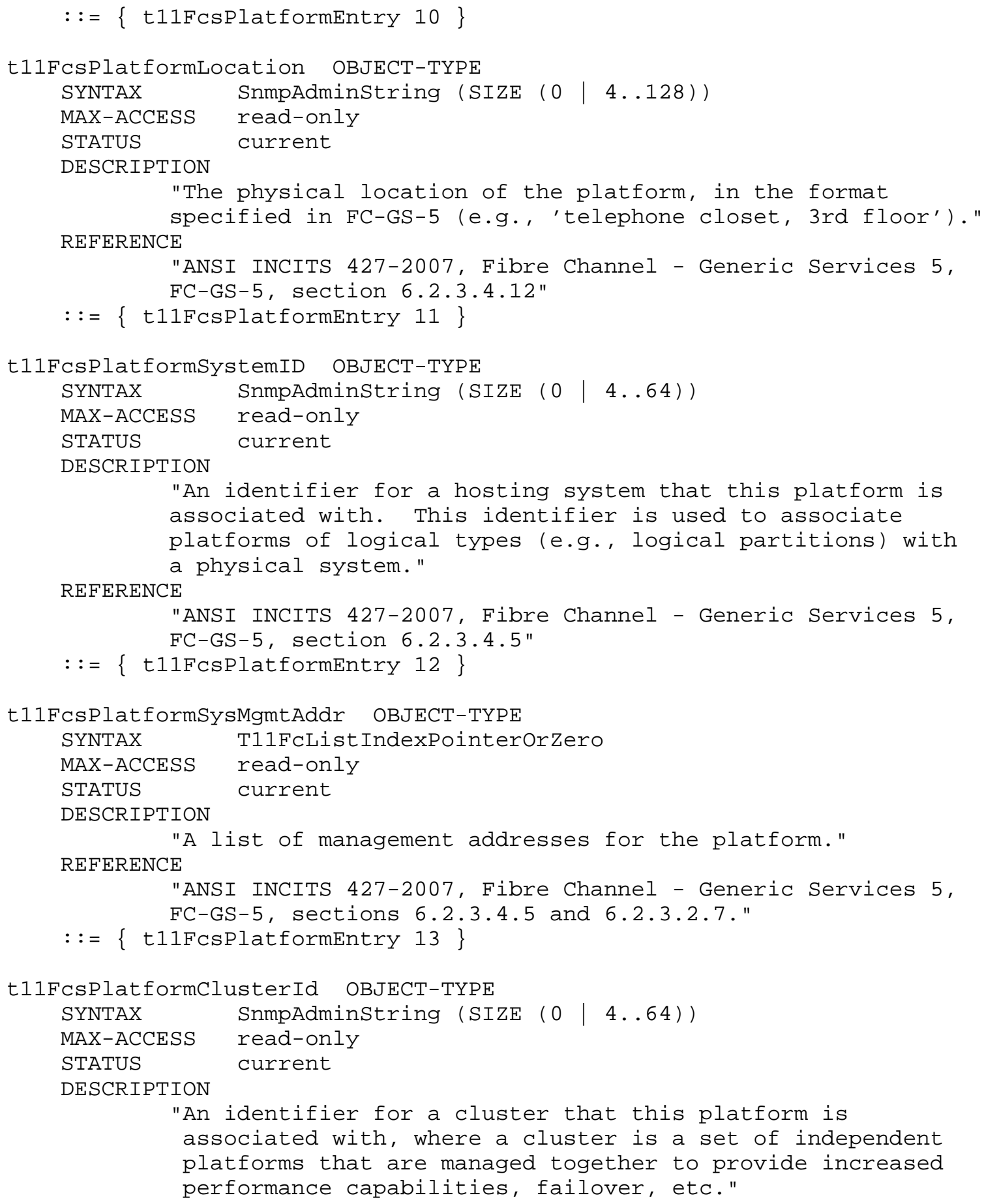




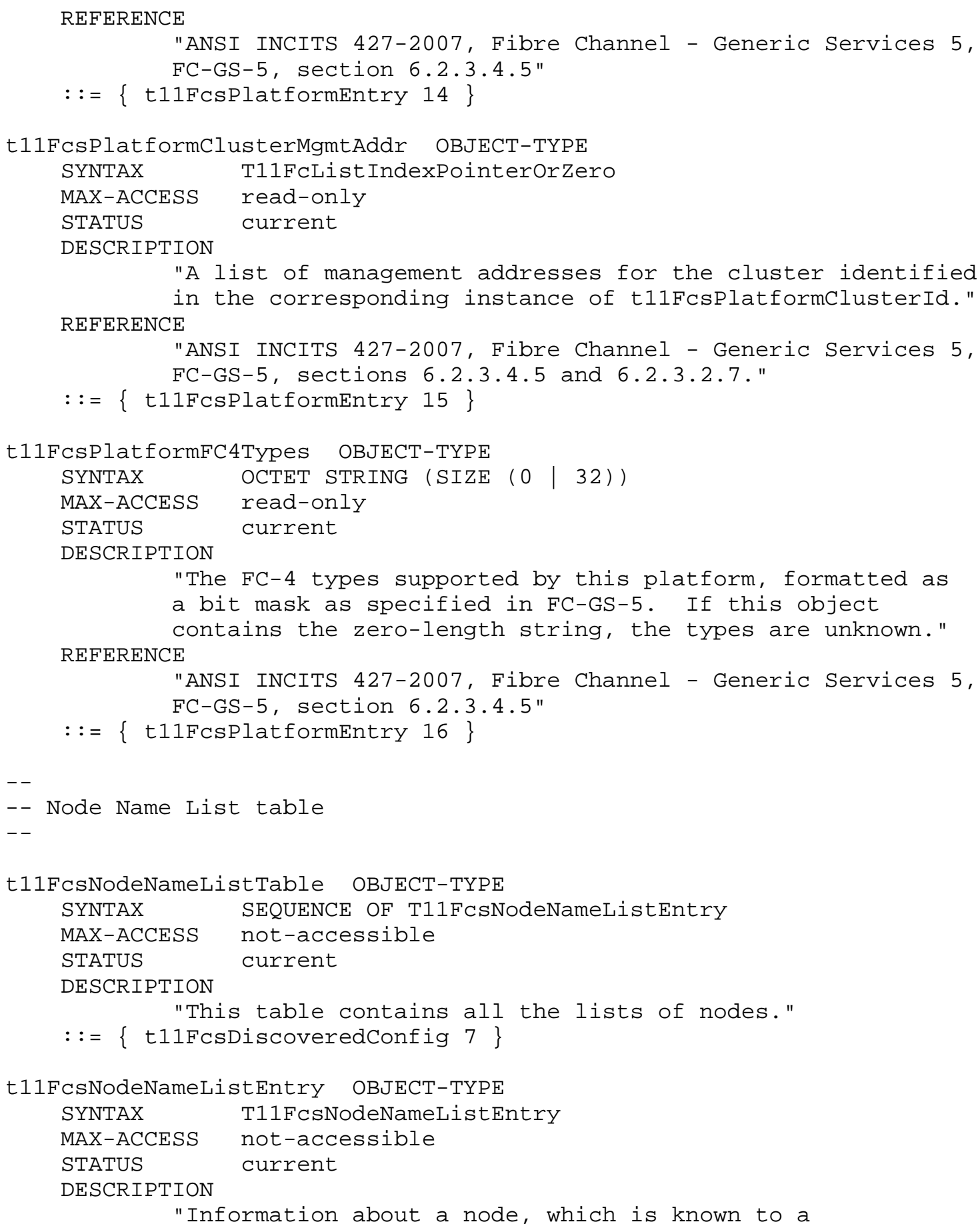




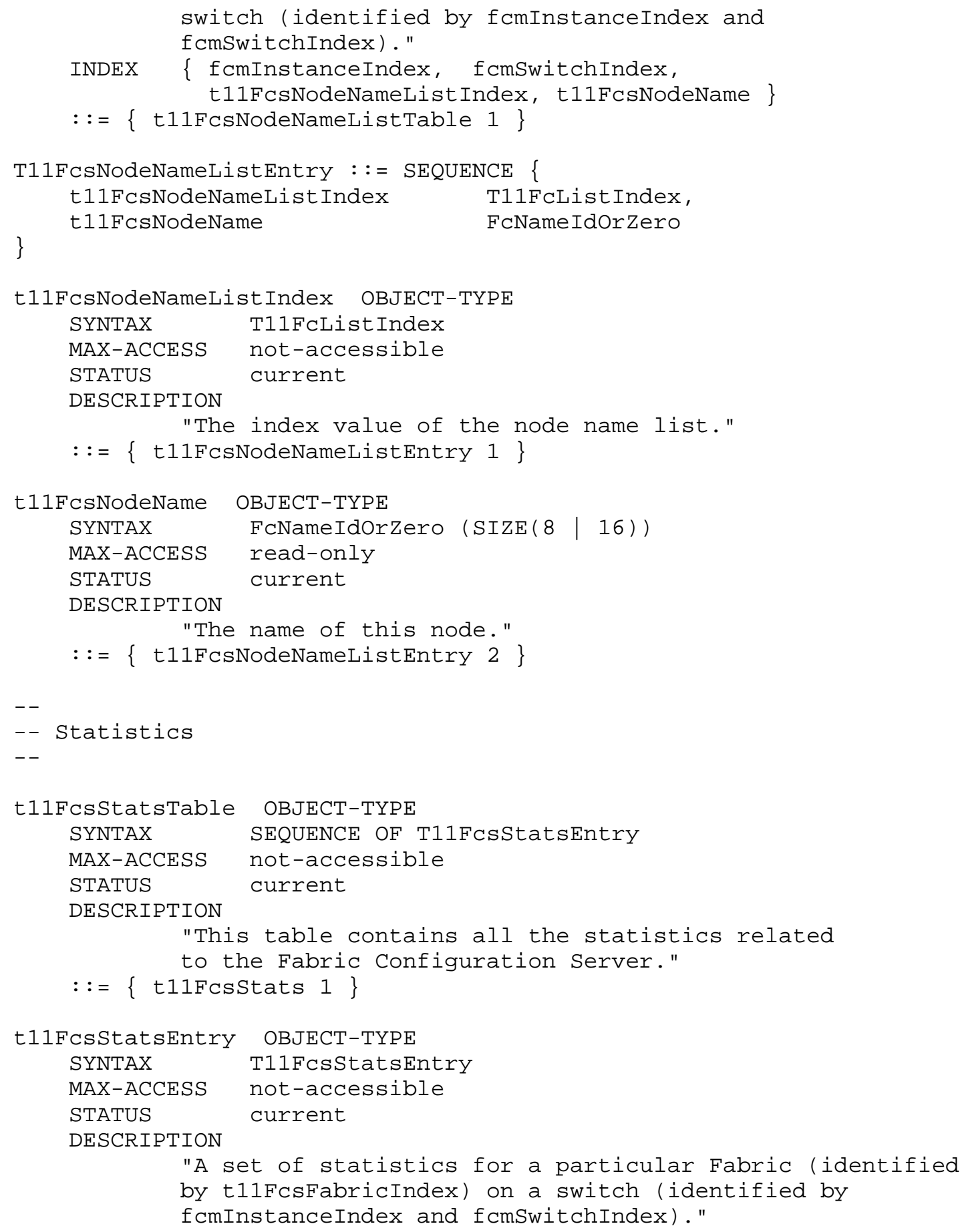




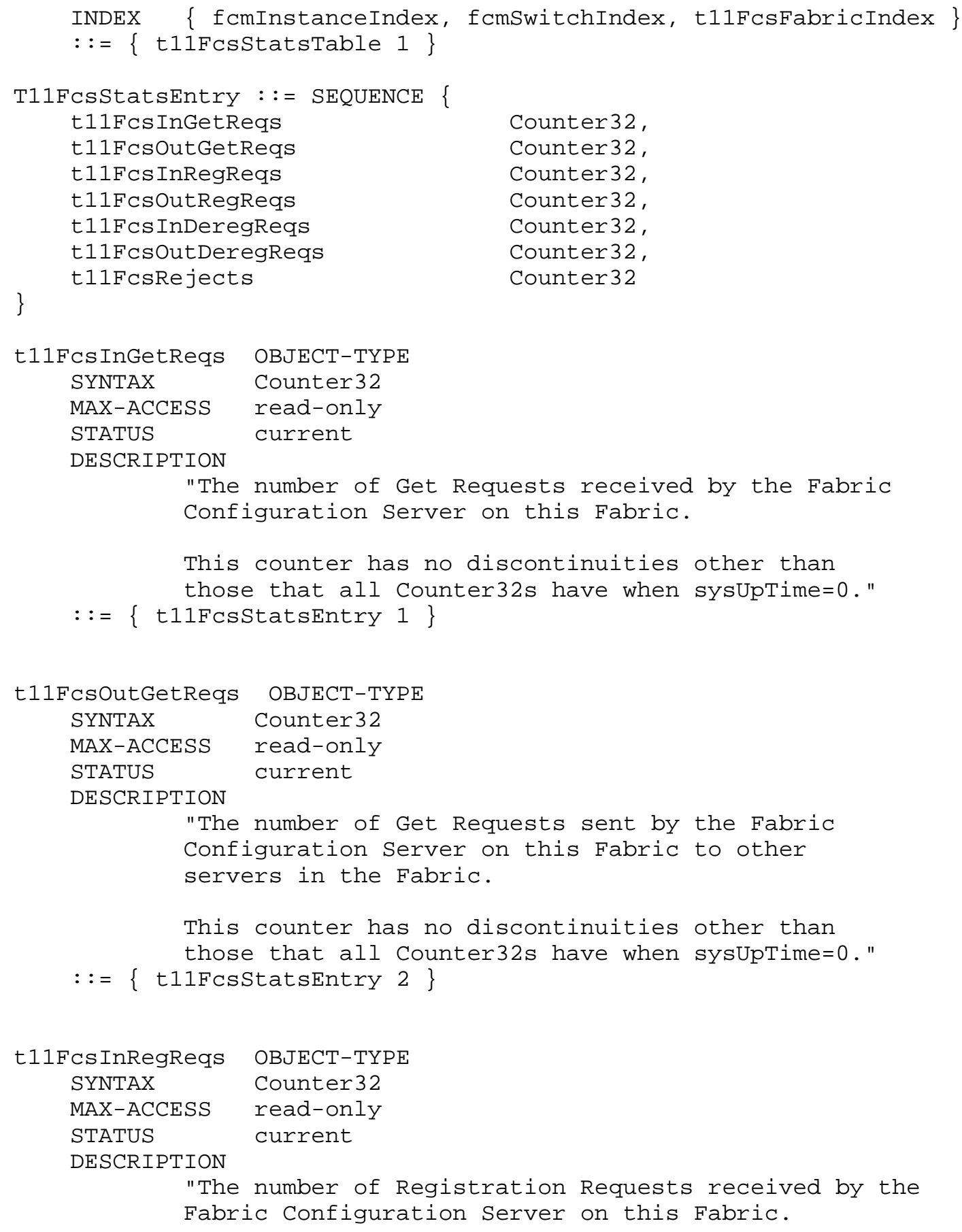




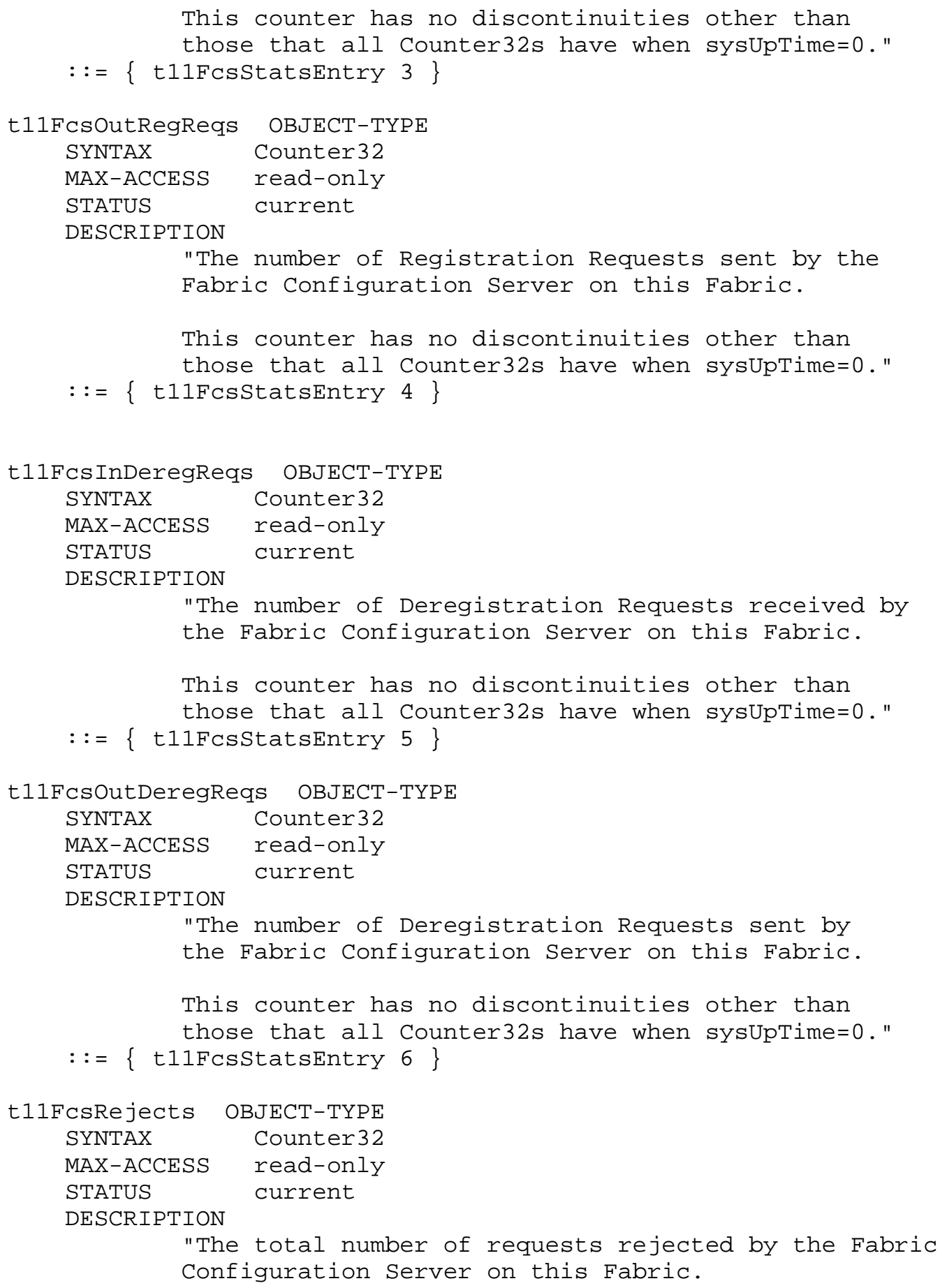




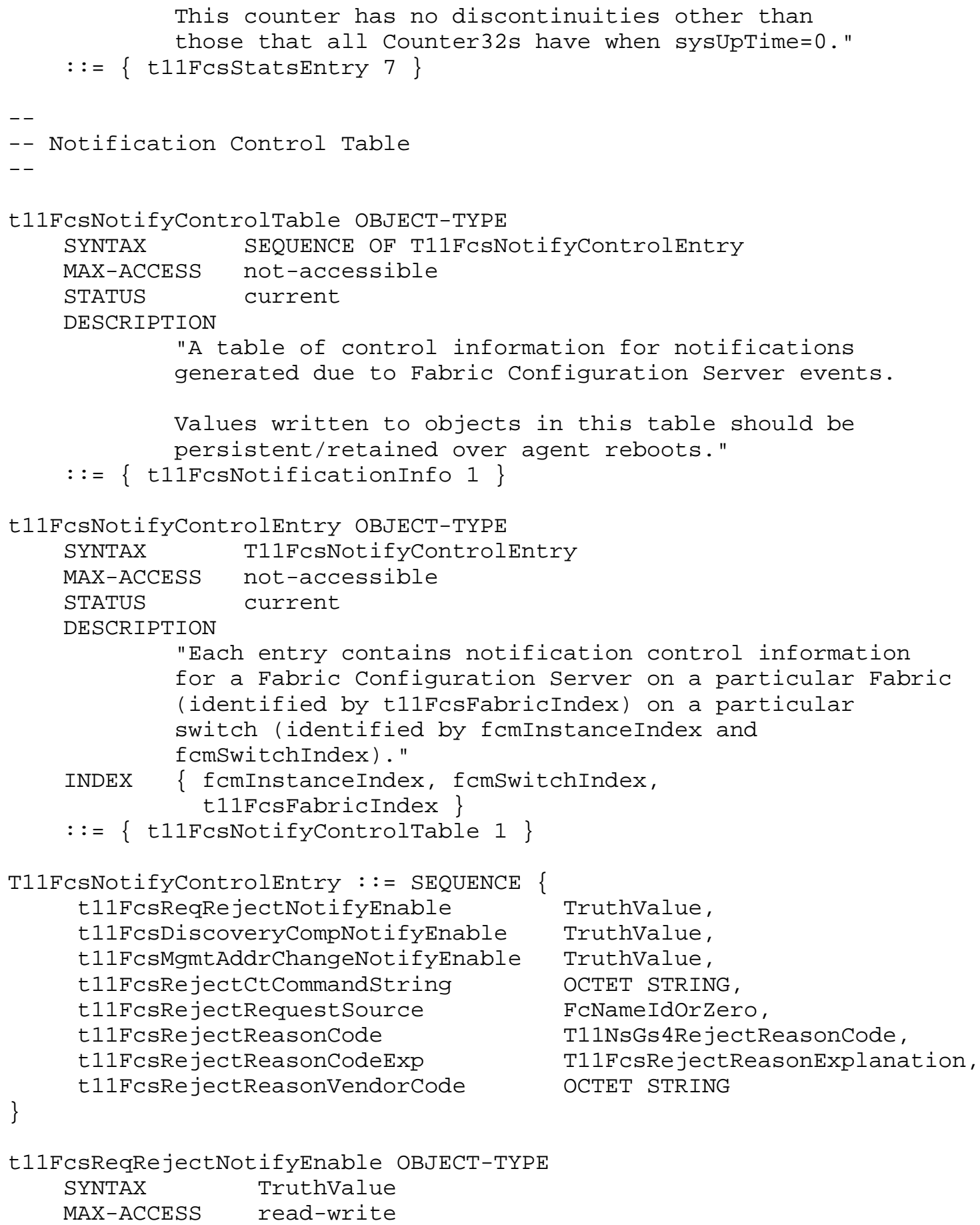




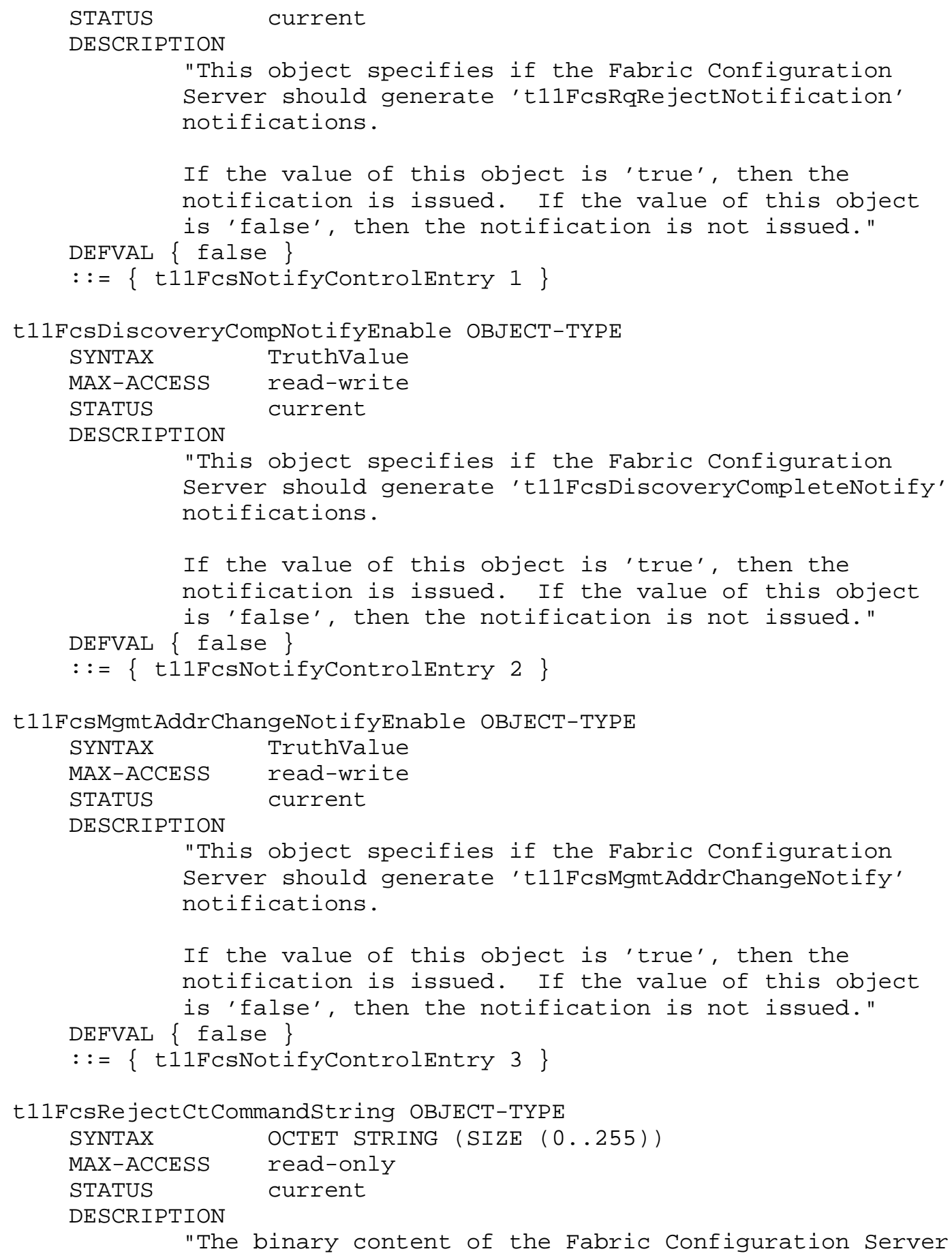




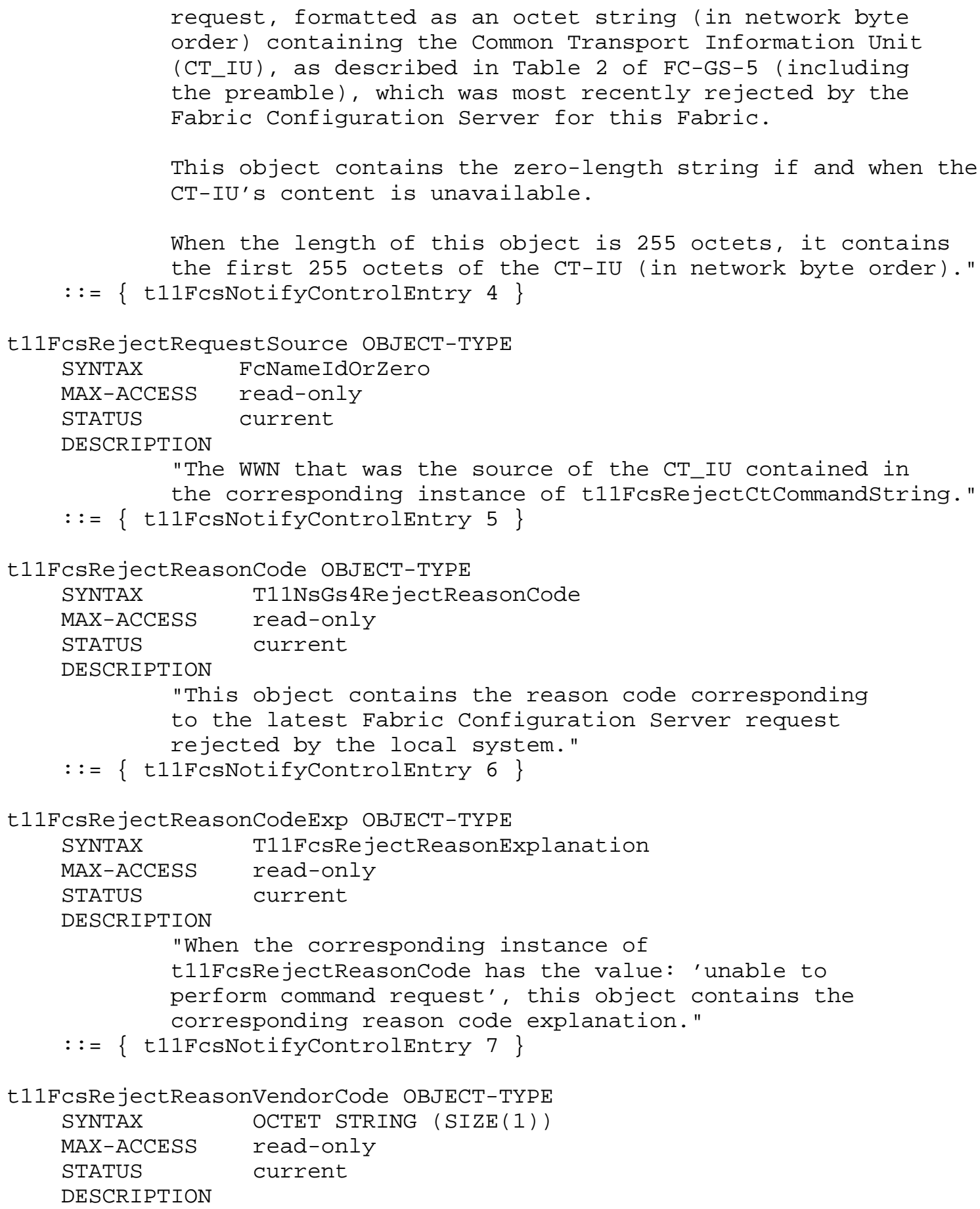




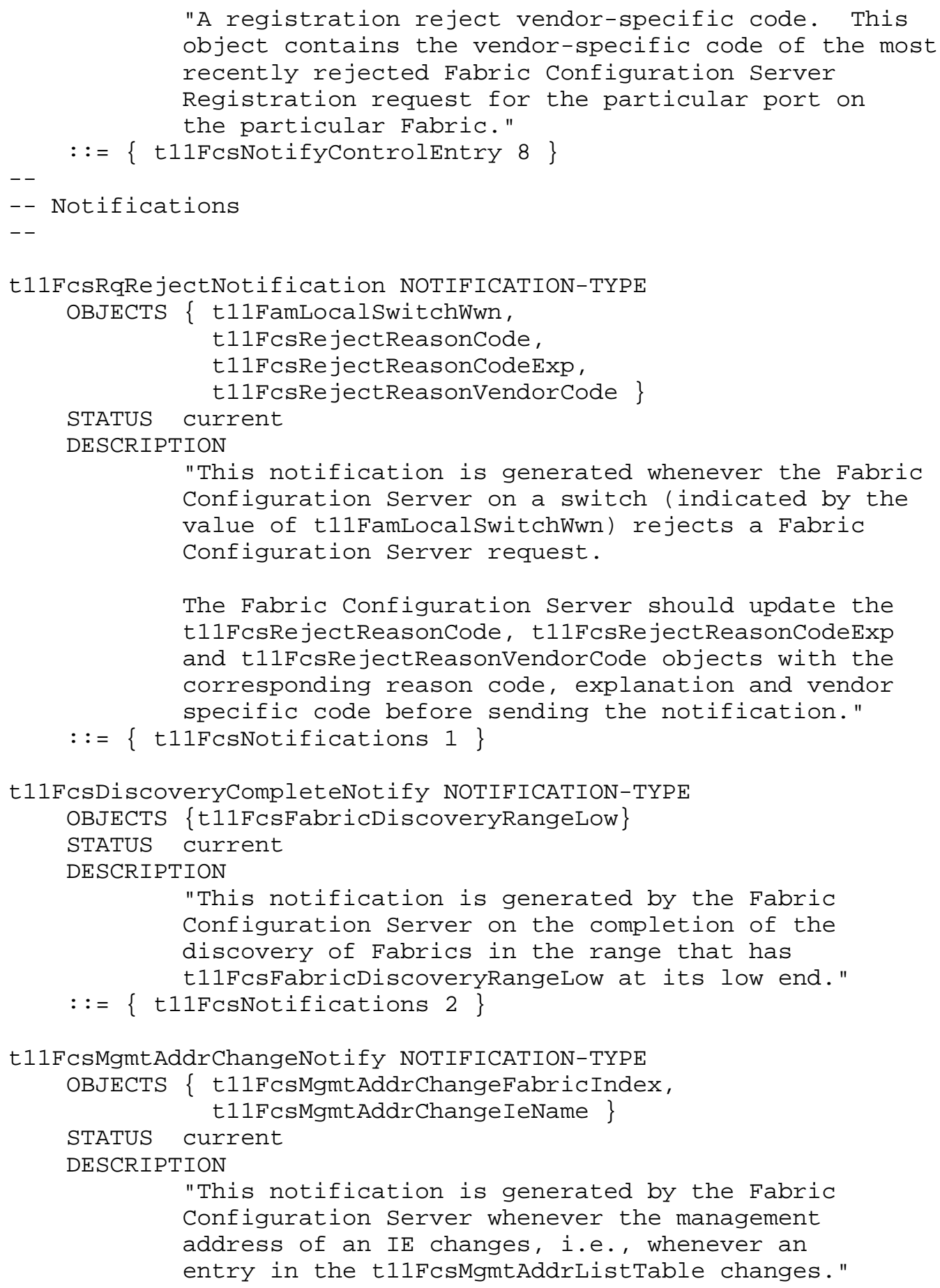


$::=\{$ t11FcsNotifications 3$\}$

t11FcsMgmtAddrChangeFabricIndex OBJECT-TYPE

SYNTAX Tl1FabricIndex

MAX-ACCESS accessible-for-notify

STATUS current

DESCRIPTION

"The index value that identifies the Fabric on which

a management address change has been detected." $::=\{$ t11FcsNotificationInfo 2$\}$

t11FcsMgmtAddrChangeIeName OBJECT-TYPE

SYNTAX FCNameIdOrZero

MAX-ACCESS accessible-for-notify

STATUS current

DESCRIPTION

"The IE for which a management address change has been detected."

$::=\{$ tilfcsNotificationInfo 3$\}$

\section{-- Conformance}

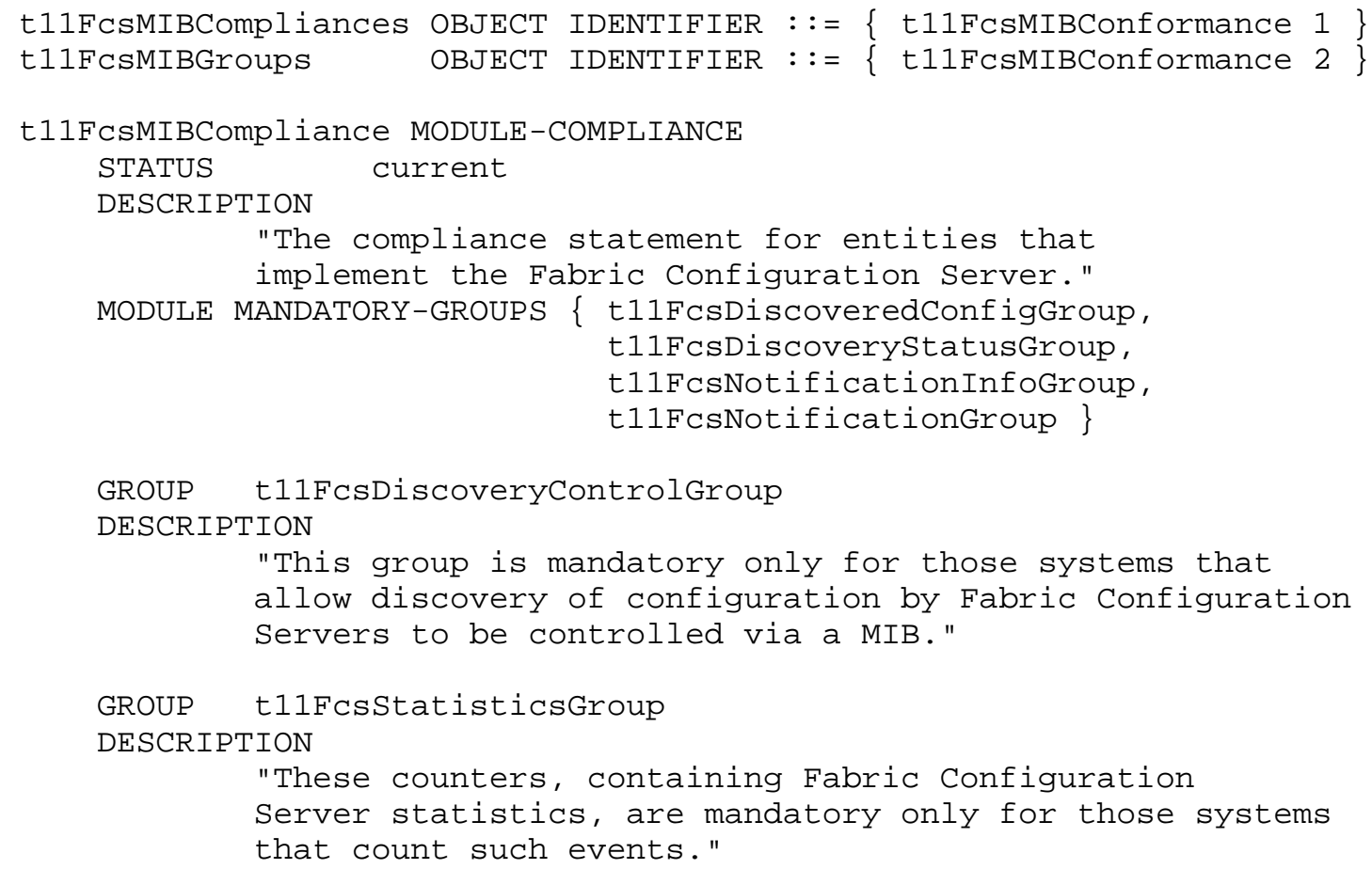




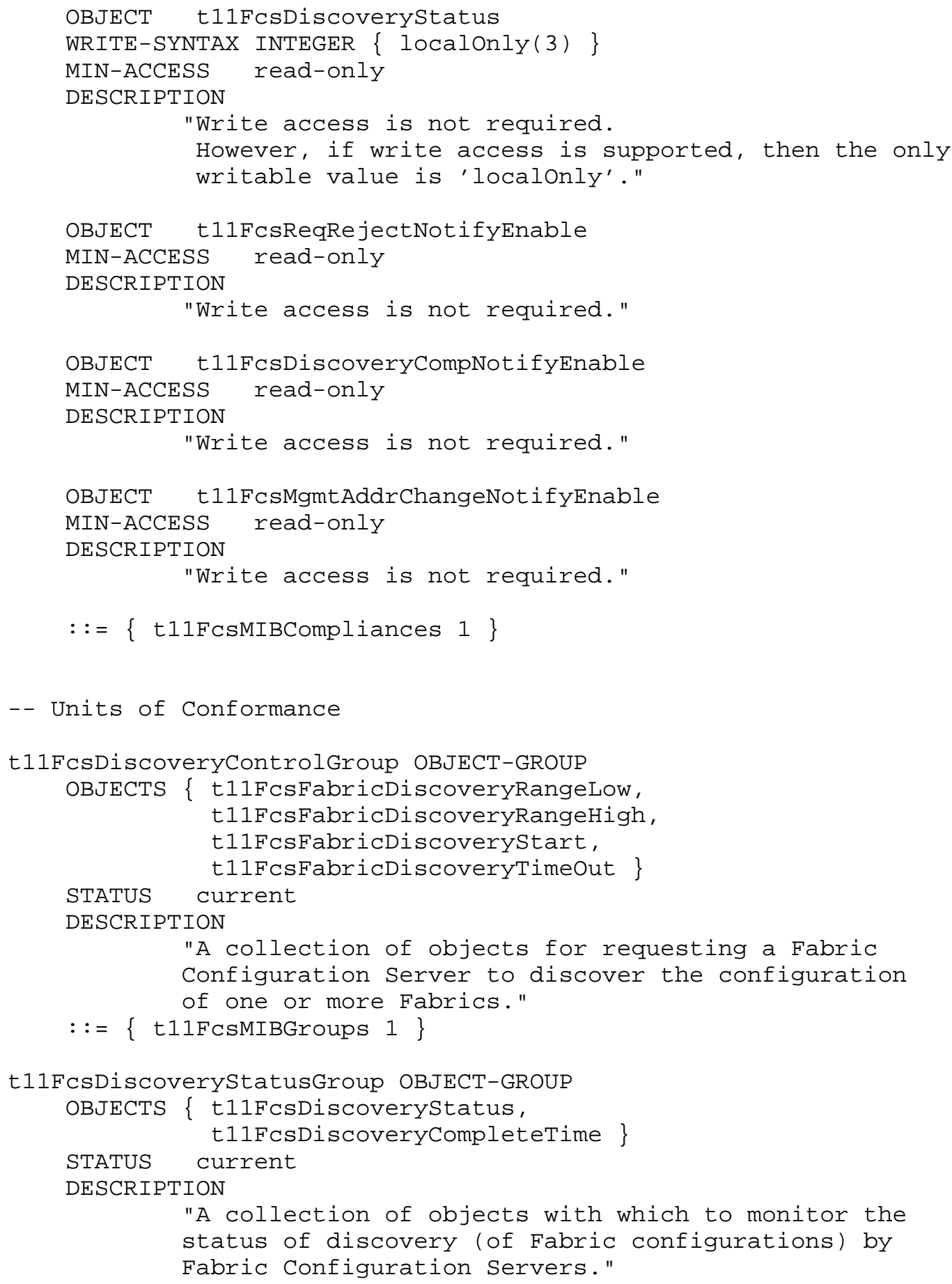


$::=\{$ t11FCsMIBGroups 2$\}$

t11FCsDiscoveredConfigGroup OBJECT-GROUP OBJECTS \{

t11FcsieType, t11FcsIeDomainId, t11Fcs IeMgmt Id, t11FcsIeFabricName, t11FcsIeLogicalName, t11Fcs IeMgmt AddrList Index, t11FcsIeInfoList, t11F csMgmtAddr, t11FcsPort Type, t11FcsportTxType, t11FcsPortModuleType, t11FcsPortPhyPortNum, t11FcsPortAttachPortNameIndex, t11Fcsportstate, t11FcsPortspeedCapab, t11FcsPortoperSpeed, t11FcsPortZoningEnfStatus, t11FcsAttachPortName, t11FcsPlatformName, t11FcsPlatformType, t11FcsPlat formNodeNameList Index, t $11 \mathrm{FcsP}$ lat formMgmtAddrList Index, t11FcsPlatformVendorId, t11FcsPlatformProduct Id, t11FcsPlatformProduct RevLevel, t11FcsPlatformDescription, t11FcsPlatformLabel, t11FcsPlatformLocation, t11FcsPlatformsystemID, t11FcsPlatformSysMgmtAddr, t11FcsPlatformclusterId, t $11 \mathrm{FcsP}$ latformClusterMgmtAddr, t11FcsPlatformFC4Types, t11FcsNodeName \}

STATUS current DESCRIPTION

"A collection of objects to contain the Fabric configuration information discovered by Fabric Configuration Servers." $::=\{$ tilfcsmiBgroups 3$\}$

t11FcstatisticsGroup OBJECT-GROUP

OBJECTS \{ t11FCsInGetReqs, t11FcsOutGetReqs, t11Fcs InRegReqs, 


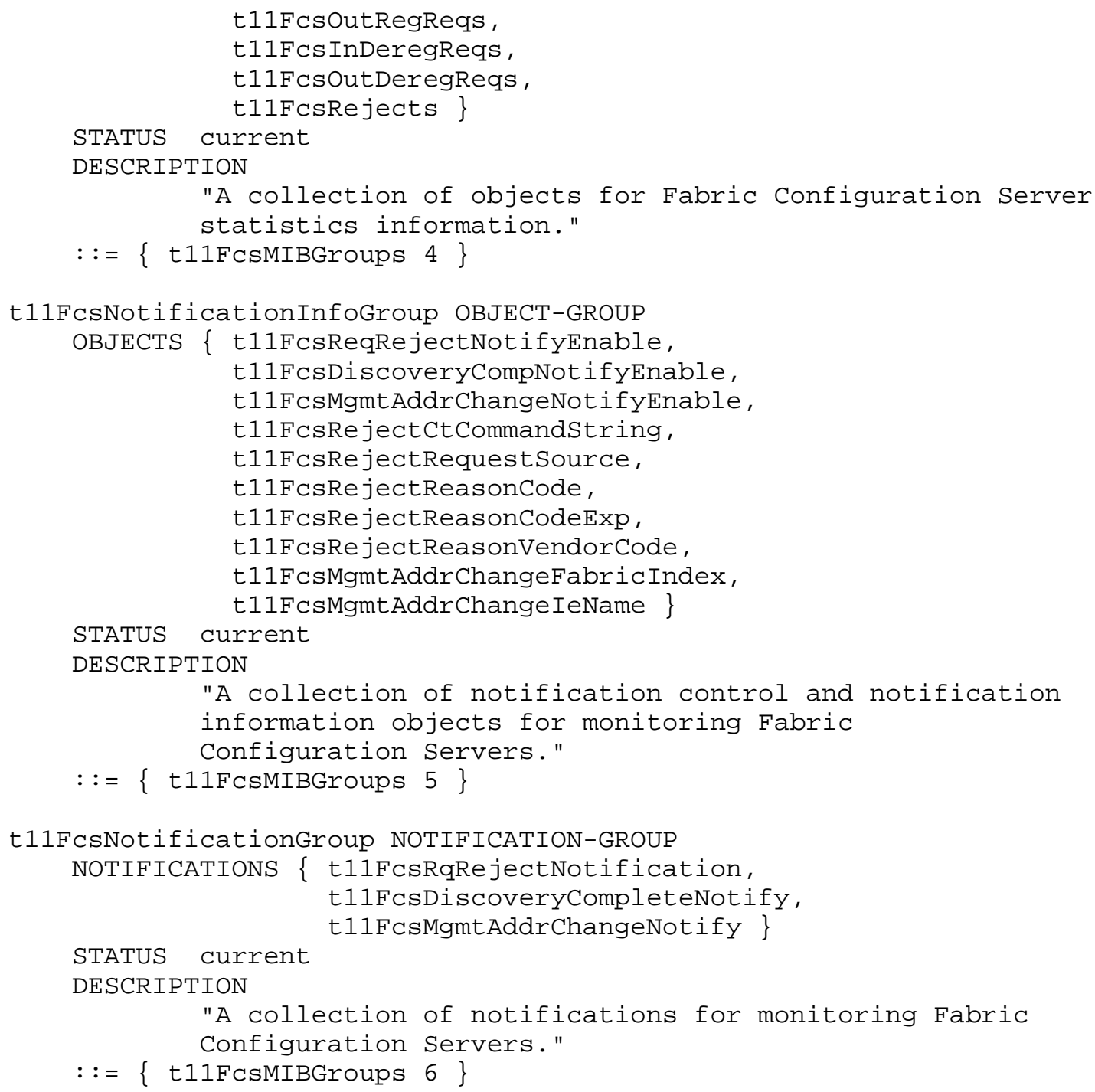

END 
7. IANA Considerations

IANA has assigned a MIB OID (162) under the mib-2 subtree.

\section{Security Considerations}

There are several management objects defined in this MIB module with a MAX-ACCESS clause of read-write and/or read-create. Such objects may be considered sensitive or vulnerable in some network environments. The support for SET operations in a non-secure environment without proper protection can have a negative effect on network operations. These objects and their sensitivity/vulnerability is:

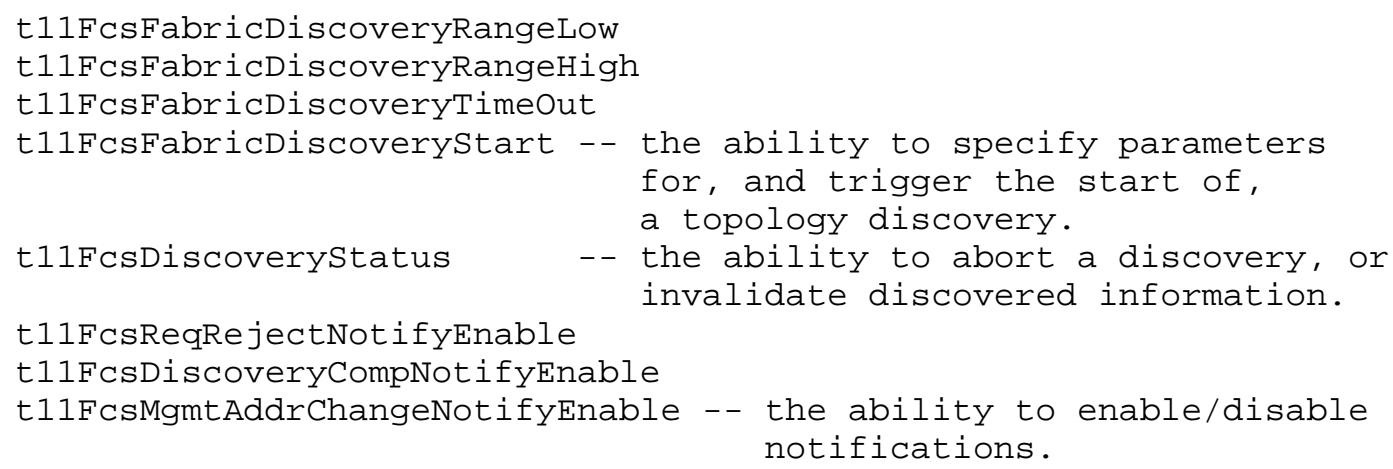

Such objects may be considered sensitive or vulnerable in some network environments. For example, the ability to invalidate discovered topology may afford an attacker the ability to hide the presence of unauthorized equipment on the network. The support for SET operations in a non-secure environment without proper protection can have a negative effect on network operations.

Some of the readable objects in this MIB module (i.e., objects with a MAX-ACCESS other than not-accessible) may be considered sensitive or vulnerable in some network environments. It is thus important to control even GET and/or NOTIFY access to these objects and possibly to even encrypt the values of these objects when sending them over the network via SNMP. These are the tables and objects and their sensitivity/vulnerability:

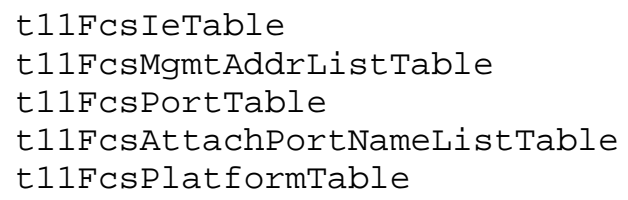




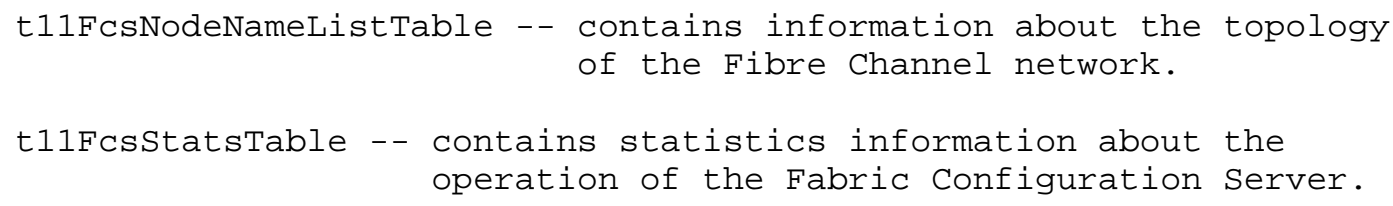

\section{Acknowledgements}

This document was originally developed and approved by the INCITS Task Group T11.5 (http://www.t11.org) as the SM-FCFGM project. We wish to acknowledge the many contributions and comments from the INCITS Technical Committee T11, especially from the following:

T11 Chair: Robert Snively, Brocade

T11 Vice Chair: Claudio DeSanti, Cisco Systems

T11.5 Chair: Roger Cummings, Symantec

T11.5 Vice Chair: Scott Kipp, McData

and T11.5 members.

The document was subsequently a work item of the IETF's IMSS Working Group, chaired by David Black (EMC Corporation). We thank Bert

Wijnen (Lucent Technologies) for his thorough review of the document. We also wish to acknowledge Dan Romascanu (Avaya), the IETF Area Director, for his comments and assistance. 
10. Normative References

[RFC2578] MCCloghrie, K., Perkins, D., Schoenwaelder, J., Case, J., Rose, M. and S. Waldbusser, "Structure of Management Information Version 2 (SMIV2)", STD 58, RFC 2578, April 1999 .

[RFC2579] McCloghrie, K., Perkins, D., Schoenwaelder, J., Case, J., Rose, M. and S. Waldbusser, "Textual Conventions for SMIV2", STD 58, RFC 2579, April 1999.

[RFC2580] McCloghrie, K., Perkins, D., Schoenwaelder, J., Case, J., Rose, M. and S. Waldbusser, "Conformance Statements for SMIV2", STD 58, RFC 2580, April 1999.

[RFC2788] Freed, N. and S. Kille, "Network Services Monitoring MIB", RFC 2788, March 2000 .

[RFC3411] Harrington, D., Presuhn, R., and B. Wijnen, "An Architecture for Describing Simple Network Management Protocol (SNMP) Management Frameworks", STD 58, RFC 3411, December 2002 .

[FC-FS] "Fibre Channel - Framing and Signaling (FC-FS)" ANSI INCITS 373-2003, http://www.t11.org/t11/stat.nsf/upnum/1331-d, April 2003.

[FC-GS-5] "Fibre Channel - Generic Services - 5 (FC-GS-5) ", ANSI INCITS 427-2007, http://www.t11.org/t11/stat.nsf/upnum/1677-d, 2007 .

[FC-SW-4] "Fibre Channel - Switch Fabric - 4 (FC-SW-4)", ANSI INCITS 418-2006, http://www.t11.org/t11/stat.nsf/upnum/1674-d, December 2006 .

[RFC4044] MCCloghrie, K., "Fibre Channel Management MIB", RFC 4044, May 2005 .

[RFC4438] DeSanti, C., Gaonkar, V., Vivek, H.K., McCloghrie, K., and S. Gai, "Fibre Channel Name Server MIB", RFC 4438, March 2006 .

[RFC4439] DeSanti, C., Gaonkar, V., McCloghrie, K., and S. Gai, "Fibre Channel Fabric Address Manager MIB", RFC 4439, March 2006 .

[RFC2119] Bradner, S., "Key words for use in RFCs to Indicate Requirement Levels", BCP 14, RFC 2119, March 1997. 
11. Informative References

[RFC2741] Daniele, M., Wijnen, B., Ellison, M., and D. Francisco, "Agent Extensibility (AgentX) Protocol Version 1", RFC 2741, January 2000 .

[RFC2837] Teow, K., "Definitions of Managed Objects for the Fabric Element in Fibre Channel Standard", RFC 2837, May 2000.

[RFC3410] Case, J., Mundy, R., Partain, D., and B. Stewart, "Introduction and Applicability Statements for InternetStandard Management Framework", RFC 3410, December 2002.

[RFC4455] Hallak-Stamler, M., Bakke, M., Lederman, Y., Krueger, M., and K. McCloghrie, "Definition of Managed objects for Small Computer System Interface (SCSI) Entities", RFC 4455, April 2006. 


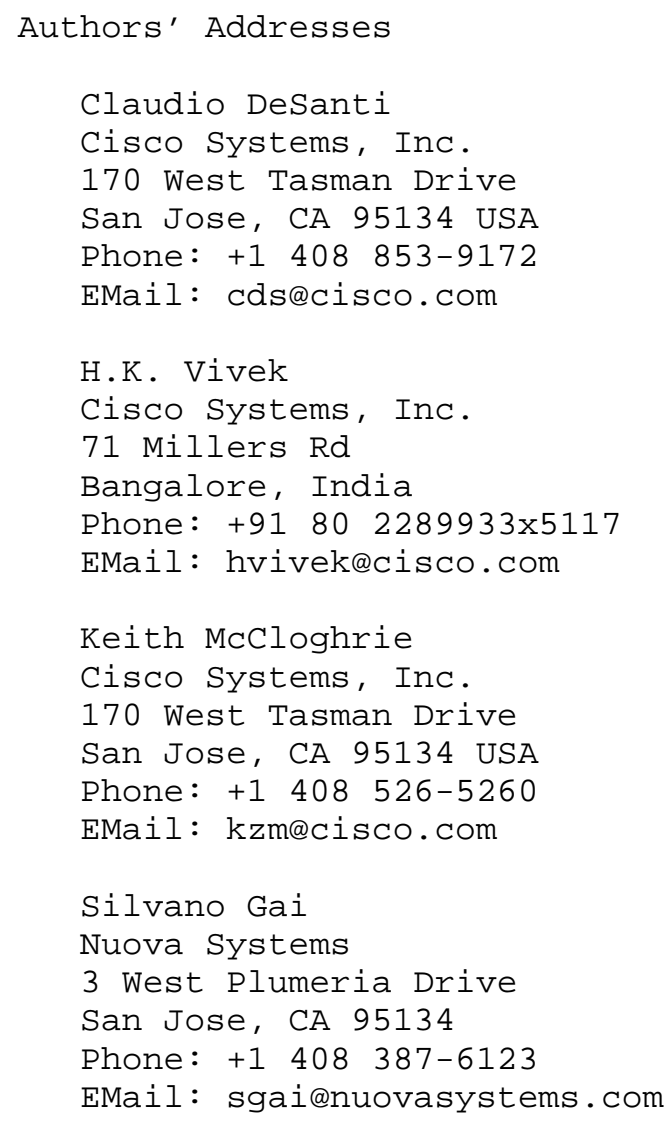


Full Copyright statement

Copyright (C) The IETF Trust (2007).

This document is subject to the rights, licenses and restrictions contained in BCP 78, and except as set forth therein, the authors retain all their rights.

This document and the information contained herein are provided on an "AS IS" basis and THE CONTRIBUTOR, THE ORGANIZATION HE/SHE REPRESENTS OR IS SPONSORED BY (IF ANY), THE INTERNET SOCIETY, THE IETF TRUST AND THE INTERNET ENGINEERING TASK FORCE DISCLAIM ALL WARRANTIES, EXPRESS OR IMPLIED, INCLUDING BUT NOT LIMITED TO ANY WARRANTY THAT THE USE OF THE INFORMATION HEREIN WILL NOT INFRINGE ANY RIGHTS OR ANY IMPLIED WARRANTIES OF MERCHANTABILITY OR FITNESS FOR A PARTICULAR PURPOSE.

Intellectual Property

The IETF takes no position regarding the validity or scope of any Intellectual Property Rights or other rights that might be claimed to pertain to the implementation or use of the technology described in this document or the extent to which any license under such rights might or might not be available; nor does it represent that it has made any independent effort to identify any such rights. Information on the procedures with respect to rights in RFC documents can be found in BCP 78 and BCP 79 .

Copies of IPR disclosures made to the IETF Secretariat and any assurances of licenses to be made available, or the result of an attempt made to obtain a general license or permission for the use of such proprietary rights by implementers or users of this specification can be obtained from the IETF on-line IPR repository at http://www.ietf.org/ipr.

The IETF invites any interested party to bring to its attention any copyrights, patents or patent applications, or other proprietary rights that may cover technology that may be required to implement this standard. Please address the information to the IETF at ietf-ipreietf.org.

Acknowledgement

Funding for the RFC Editor function is currently provided by the Internet Society. 